\title{
Totally symmetric functions are reconstructible from identification minors
}

\author{
Erkko Lehtonen* \\ Computer Science and Communications Research Unit \\ University of Luxembourg \\ Luxembourg, Luxembourg \\ erkko@campus.ul.pt
}

Submitted: Nov 1, 2012; Accepted: Mar 25, 2014; Published: Apr 16, 2014

Mathematics Subject Classifications: 08A40, 05E05, 05C60

\begin{abstract}
We formulate a reconstruction problem for functions of several arguments: Is a function of several arguments uniquely determined, up to equivalence, by its identification minors? We establish some positive and negative results on this reconstruction problem. In particular, we show that totally symmetric functions (of sufficiently large arity) are reconstructible.
\end{abstract}

Keywords: reconstruction problem; function of several arguments; totally symmetric function

\section{Introduction}

Reconstruction problems have received great attention over the past decades. In very abstract terms, a reconstruction problem asks whether a mathematical object can be recovered from partial information. The reconstruction problems we discuss here fall under the following general framework: given a class of mathematical objects and a way of deriving subobjects of a given object, we consider the question whether an object is uniquely determined, up to some kind of isomorphism, by its collection of subobjects.

Perhaps the most famous reconstruction problem is the following: Can every graph with at least three vertices be reconstructed, up to isomorphism, from its collection of one-vertex-deleted subgraphs? It was conjectured by Kelly [17] and Ulam [34] that the answer is positive. The conjecture has been verified by computer for graphs with at most 11 vertices (McKay [25]), and it has been proved for several infinite classes of graphs, such

\footnotetext{
*Current affiliation: Centro de Álgebra da Universidade de Lisboa, Lisbon, Portugal.
} 
as trees (Kelly [18]), regular graphs, disconnected graphs, and so on. We refer the reader to survey articles, textbooks, and reference books [1, 3, 4, 5, 16, 23, 28] for further details and references.

The reconstruction problem stated above can be varied in several ways. For example, we might consider the collection of subgraphs formed by deleting edges instead of vertices (see Harary [15] and Ellingham [13]), or we could consider directed graphs (infinite nonreconstructible families have been constructed by Stockmeyer [32]) or hypergraphs (infinite nonreconstructible families have been constructed by Kocay [19]). Analogous reconstruction problems have been formulated also for other kinds of mathematical objects, such as relations (see Fraïssé [14]), posets (see the survey by Rampon [30]), matrices (see Manvel and Stockmeyer [24]), matroids (see Brylawski [7, 8]), and integer partitions (see Monks [26]).

In this paper we formulate a reconstruction problem for functions of several arguments. We shall take as the derived objects of a function $f: A^{n} \rightarrow B$ its identification minors, i.e., functions obtained from $f$ by identifying a pair of its arguments. The notion of isomorphism is based on the equivalence relation that relates two $n$-ary functions if and only if each one can be obtained from the other by permutation of arguments. The reconstruction problem can thus be stated as follows: Can a function $f: A^{n} \rightarrow B$ be reconstructed, up to equivalence, from its identification minors?

The purpose of this paper is to take some first steps towards providing an answer to this question. A necessary condition for a positive answer is that the arity of the function must be sufficiently large. Indeed, as will be explained in Remark 3.2, if $n \leqslant|A|$, then no function $f: A^{n} \rightarrow B$ is reconstructible; this also shows that functions defined on infinite domains are not reconstructible. Furthermore, Example 3.13 shows that there exist functions $f: A^{n} \rightarrow B$ with $n=|A|+1$ that are not reconstructible. We will obtain several other results, both positive and negative, about the reconstructibility of functions. The main result is Theorem 5.1 which asserts that totally symmetric functions (of sufficiently large arity) are reconstructible.

Identification minors and equivalence of functions are related in an essential way to a quasiordering of functions, the so-called minor relation, which is defined as follows: a function $f: A^{n} \rightarrow B$ is a minor of another function $g: A^{m} \rightarrow B$, if there exists a map $\sigma:\{1, \ldots, m\} \rightarrow\{1, \ldots, n\}$ such that $f\left(a_{1}, \ldots, a_{n}\right)=g\left(a_{\sigma(1)}, \ldots, a_{\sigma(m)}\right)$ for all $\left(a_{1}, \ldots, a_{n}\right) \in A^{n}$. The reconstruction problem is thus a deep and intriguing question about the structure of the minor ordering of functions. Minors have been widely studied in the literature, under different names. Minors are called "identification minors" by Ekin, Foldes, Hammer, and Hellerstein [12], "I $\mathcal{I}$-minors" (where $\mathcal{I}$ stands for the set containing just the identity function on $A$ ) by Pippenger [29], "subfunctions" by Zverovich [37], "functions obtained by simple variable substitution" by Couceiro and Foldes [9], " $\mathcal{J}$ subfunctions" (where $\mathcal{J}$ stands for the clone of projections on $A$ ) by Lehtonen [21], " $\mathcal{P}_{A^{-}}$ minors" (where $\mathcal{P}_{A}$ stands for the clone of projections on $A$ ) by Lehtonen and Szendrei [22], and "simple minors" by Couceiro and Lehtonen [10].

This paper is organised as follows. In Section 2, we provide basic definitions on functions and identification minors that will be needed in the sequel, and we recall the 
definition of multiset. In Section 3, we formulate a reconstruction problem for functions and identification minors, and we recall the usual terminology of reconstruction problems in the current setting. We present some examples of reconstructible and recognizable classes of functions, as well as examples of nonreconstructible functions and reconstructible parameters. One of our first results is that functions determined by supp or oddsupp (of sufficiently large arity) are reconstructible. In Section 4, we discuss functions with a unique identification minor, and we establish an auxiliary result that asserts that 2-set-transitive functions have a unique identification minor. This will find an application in Section 5 , where we prove our main result that totally symmetric functions (of sufficiently large arity) are reconstructible. In Section 6, we translate the reconstruction problem of functions and identification minors into the language of graphs and hypergraphs. Occasionally throughout the paper, we present open problems to indicate directions for future work.

\section{Preliminaries}

\subsection{General}

Throughout this paper, we let $k, m$ and $n$ be positive integers, and we let $A$ and $B$ be arbitrary sets with at least two elements. For reasons that will become clear in Remark 3.2, we may assume that these sets are finite, and $k$ usually stands for the cardinality $|A|$ of $A$. For a positive integer $n$, we denote the set $\{1, \ldots, n\}$ by $[n]$. We denote the set of all 2-element subsets of $[n]$ by $\left(\begin{array}{c}{[n]} \\ 2\end{array}\right)$. We denote tuples by bold-face letters and components of a tuple by the corresponding italic letters with subscripts, e.g., $\mathbf{a}=\left(a_{1}, \ldots, a_{n}\right)$. We reserve the symbol $\mathbf{k}$ to denote the $k$-tuple $(1,2, \ldots, k) \in[k]^{k}$.

Let $\mathbf{a} \in A^{n}$, and let $\sigma:[m] \rightarrow[n]$. We write $\mathbf{a} \sigma$ to denote the $m$-tuple $\left(a_{\sigma(1)}, \ldots, a_{\sigma(m)}\right)$. Since the $n$-tuple a can be formally seen as a map a: $[n] \rightarrow A$, the $m$-tuple a $\sigma$ is just the composite map a $\circ \sigma:[m] \rightarrow A$. It is worth stressing here that we always compose functions and permutations right-to-left, so $\sigma \tau$ or $\sigma \circ \tau$ means "do $\tau$ first, then do $\sigma$ ".

\subsection{Functions of several arguments and identification minors}

A function (of several arguments) from $A$ to $B$ is a map $f: A^{n} \rightarrow B$ for some positive integer $n$, called the arity of $f$. Functions of several arguments from $A$ to $A$ are called operations on $A$. Operations on $\{0,1\}$ are called Boolean functions. We denote the set of all $n$-ary functions from $A$ to $B$ by $\mathcal{F}_{A B}^{(n)}$, and we denote the set of all functions from $A$ to $B$ of any finite arity by $\mathcal{F}_{A B}$; in other words, $\mathcal{F}_{A B}^{(n)}=B^{A^{n}}$ and $\mathcal{F}_{A B}=\bigcup_{n \geqslant 1} \mathcal{F}_{A B}^{(n)}$. For any $\mathcal{C} \subseteq \mathcal{F}_{A B}$, we denote $\mathcal{C}^{(n)}:=\mathcal{C} \cap \mathcal{F}_{A B}^{(n)}$; this is called the $n$-ary part of $\mathcal{C}$.

A function $f: A^{n} \rightarrow B$ is totally symmetric if for every permutation $\sigma$ of $[n]$ it holds that $f(\mathbf{a})=f(\mathbf{a} \sigma)$ for all $\mathbf{a} \in A^{n}$.

For integers $n$ and $i$ such that $1 \leqslant i \leqslant n$, the $i$-th $n$-ary projection on $A$ is the operation $\operatorname{pr}_{i}^{(n)}: A^{n} \rightarrow A$ given by the rule $\operatorname{pr}_{i}^{(n)}\left(a_{1}, \ldots, a_{n}\right)=a_{i}$ for all $\left(a_{1}, \ldots, a_{n}\right) \in A^{n}$.

Let $f: A^{n} \rightarrow B$. For $i \in[n]$, the $i$-th argument of $f$ is essential, or $f$ depends on the 
$i$-th argument, if there exist elements $\mathbf{a}, \mathbf{b} \in A^{n}$ such that $a_{j}=b_{j}$ for all $j \in[n] \backslash\{i\}$ and $f(\mathbf{a}) \neq f(\mathbf{b})$. Arguments that are not essential are inessential.

We say that a function $f: A^{n} \rightarrow B$ is a minor of another function $g: A^{m} \rightarrow B$, and we write $f \leqslant g$, if there exists a map $\sigma:[m] \rightarrow[n]$ such that $f(\mathbf{a})=g(\mathbf{a} \sigma)$ for all $\mathbf{a} \in A^{m}$. The minor relation $\leqslant$ is a quasiorder on $\mathcal{F}_{A B}$, and, as for all quasiorders, it induces an equivalence relation on $\mathcal{F}_{A B}$ by the following rule: $f \equiv g$ if and only if $f \leqslant g$ and $g \leqslant f$. We say that $f$ and $g$ are equivalent if $f \equiv g$. Furthermore, $\leqslant$ induces a partial order on the quotient $\mathcal{F}_{A B} / \equiv$. (Informally speaking, $f$ is a minor of $g$, if $f$ can be obtained from $g$ by permutation of arguments, addition of inessential arguments, deletion of inessential arguments, and identification of arguments. If $f$ and $g$ are equivalent, then each one can be obtained from the other by permutation of arguments, addition of inessential arguments, and deletion of inessential arguments.) We will often distinguish between functions only up to equivalence, i.e., we are dealing with the 三-classes of functions. We denote the $\equiv$-class of $f$ by $f / \equiv$. Note that equivalent functions have the same number of essential arguments and every nonconstant function is equivalent to a function with no inessential arguments. Note also in particular that if $f, g: A^{n} \rightarrow B$, then $f \equiv g$ if and only if there exists a bijection $\sigma:[n] \rightarrow[n]$ such that $f(\mathbf{a})=g(\mathbf{a} \sigma)$ for all $\mathbf{a} \in A^{n}$.

A set $\mathcal{C} \subseteq \mathcal{F}_{A B}$ of functions is closed under formation of minors if for all $f, g \in \mathcal{F}_{A B}$, the conditions $f \leqslant g$ and $g \in \mathcal{C}$ together imply $f \in \mathcal{C}$. All clones are closed under formation of minors. (Recall that a clone on $A$ is a set of operations on $A$ that contains all projections and is closed under functional composition; see [11, 20,33].) A characterization of sets of functions closed under formation of minors in terms of a Galois connection between functions and so-called constraints was presented by Pippenger [29] for functions with finite domains, and this result was later extended to functions with arbitrary domains by Couceiro and Foldes [9].

Of particular interest to us are the following minors. Let $n \geqslant 2$, and let $f: A^{n} \rightarrow B$. For each $I \in\left(\begin{array}{c}{[n]} \\ 2\end{array}\right)$, we define the function $f_{I}: A^{n-1} \rightarrow B$ by the rule $f_{I}(\mathbf{a})=f\left(\mathbf{a} \delta_{I}\right)$ for all $\mathbf{a} \in A^{n-1}$, where $\delta_{I}:[n] \rightarrow[n-1]$ is defined as

$$
\delta_{I}(i)= \begin{cases}i, & \text { if } i<\max I, \\ \min I, & \text { if } i=\max I, \\ i-1, & \text { if } i>\max I\end{cases}
$$

In other words, if $I=\{i, j\}$ with $i<j$, then

$$
f_{I}\left(a_{1}, \ldots, a_{n-1}\right)=f\left(a_{1}, \ldots, a_{j-1}, a_{i}, a_{j}, \ldots, a_{n-1}\right) .
$$

Note that $a_{i}$ occurs twice on the right side of the above equality: both at the $i$-th and at the $j$-th position. We will refer to the function $f_{I}$ as an identification minor of $f$. This name is motivated by the fact that $f_{I}$ is obtained from $f$ by identifying the arguments indexed by the couple $I$.

Example 2.1. Let $f: \mathbb{R}^{4} \rightarrow \mathbb{R}$ be given by $f\left(x_{1}, x_{2}, x_{3}, x_{4}\right)=x_{1}^{2} x_{2}-x_{1} x_{3}+2 x_{3} x_{4}$. The identification minors of $f$ are

$$
f_{\{1,2\}}\left(x_{1}, x_{2}, x_{3}\right)=x_{1}^{3}-x_{1} x_{2}+2 x_{2} x_{3}, \quad f_{\{2,3\}}\left(x_{1}, x_{2}, x_{3}\right)=x_{1}^{2} x_{2}-x_{1} x_{2}+2 x_{2} x_{3},
$$




$$
\begin{array}{lll}
f_{\{1,3\}}\left(x_{1}, x_{2}, x_{3}\right)=x_{1}^{2} x_{2}-x_{1}^{2}+2 x_{1} x_{3}, & & f_{\{2,4\}}\left(x_{1}, x_{2}, x_{3}\right)=x_{1}^{2} x_{2}-x_{1} x_{3}+2 x_{2} x_{3}, \\
f_{\{1,4\}}\left(x_{1}, x_{2}, x_{3}\right)=x_{1}^{2} x_{2}+x_{1} x_{3}, & & f_{\{3,4\}}\left(x_{1}, x_{2}, x_{3}\right)=x_{1}^{2} x_{2}-x_{1} x_{3}+2 x_{3}^{2} .
\end{array}
$$

Example 2.2. Let $g:\{0,1\}^{3} \rightarrow\{0,1\}$ be given by $g\left(x_{1}, x_{2}, x_{3}\right)=x_{1} x_{2}+x_{2} x_{3}$ (addition and multiplication modulo 2). The identification minors of $g$ are

$$
g_{\{1,2\}}\left(x_{1}, x_{2}\right)=x_{1}+x_{1} x_{2}, \quad g_{\{1,3\}}\left(x_{1}, x_{2}\right)=0, \quad g_{\{2,3\}}\left(x_{1}, x_{2}\right)=x_{1} x_{2}+x_{2} .
$$

Note that $g_{\{1,2\}} \equiv g_{\{2,3\}}$.

Example 2.3. Let $n$ be an integer at least 2 , let $A:=\{1, \ldots n\}$, let $B$ be a set with at least two elements, and let $\alpha$ and $\beta$ be distinct elements of $B$. Define the function $h: A^{n} \rightarrow B$ by the rule

$$
h\left(a_{1}, \ldots, a_{n}\right)= \begin{cases}\alpha, & \text { if }\left(a_{1}, \ldots, a_{n}\right)=(1, \ldots, n), \\ \beta, & \text { otherwise }\end{cases}
$$

It is clear that $h$ depends on all of its arguments, and for every $I \in\left(\begin{array}{c}{[n]} \\ 2\end{array}\right)$, the identification minor $h_{I}: A^{n-1} \rightarrow B$ is the constant map taking value $\beta$.

\subsection{Multisets}

Let $\mathbb{N}:=\{0,1,2, \ldots\}$. A finite multiset $S$ on a set $X$ is a couple $\left(X, \mathbf{1}_{S}\right)$, where $\mathbf{1}_{S}: X \rightarrow$ $\mathbb{N}$ is a map with the property that the set $\left\{x \in X: \mathbf{1}_{S}(x) \neq 0\right\}$ is finite. Then the sum $\sum_{x \in X} \mathbf{1}_{S}(x)$ is a well-defined natural number, and it is called the cardinality of $S$. The map $\mathbf{1}_{S}$ is called a multiplicity function, and for each $x \in X$, the number $\mathbf{1}_{S}(x)$ is called the multiplicity of $x$ in $S$. If $\mathbf{1}_{S}(x)>0$, then $x$ is called an element of $S$. We denote the set of all finite multisets on $X$ by $\mathcal{M}(X)$, and we denote the set of all finite multisets of cardinality $n$ on $X$ by $\mathcal{M}_{n}(X)$.

We may represent a finite multiset $S$ as a list enclosed in angle brackets, where each element $x \in X$ occurs $\mathbf{1}_{S}(x)$ times, e.g., $\langle 0,0,0,1,1,2\rangle$. Also, if $\left(a_{i}\right)_{i \in I}$ is a finite indexed family of elements of $X$, then we will write $\left\langle a_{i}: i \in I\right\rangle$ to denote the multiset in which the multiplicity of each $x \in X$ equals $\left|\left\{i \in I: a_{i}=x\right\}\right|$.

Let $S$ and $T$ be finite multisets over $X$. The multiset sum $S \uplus T$ and the difference $S \backslash T$ of $S$ and $T$ are finite multisets on $X$ defined by the multiplicity functions

$$
\begin{gathered}
\mathbf{1}_{S \uplus T}(x)=\mathbf{1}_{S}(x)+\mathbf{1}_{T}(x), \\
\mathbf{1}_{S \backslash T}(x)=\max \left(\mathbf{1}_{S}(x)-\mathbf{1}_{T}(x), 0\right) .
\end{gathered}
$$

If $S$ is a multiset on $X$, then we write $\operatorname{set}(S)$ to denote the set $\left\{x \in X: \mathbf{1}_{S}(x) \neq 0\right\}$, called the underlying set of $S$. 


\section{Reconstructing functions from identification minors}

\subsection{Reconstruction problem for functions of several arguments}

We recall the usual terminology of reconstruction problems in the setting of functions of several arguments and identification minors. Assume that $n \geqslant 2$ and let $f: A^{n} \rightarrow B$.

(i) The deck of $f$, denoted $\operatorname{deck} f$, is the multiset $\left\langle f_{I} / \equiv: I \in\left(\begin{array}{c}{[n]} \\ 2\end{array}\right)\right\rangle$ of the equivalence classes of the identification minors of $f$. Any element of the deck of $f$ is called a card of $f$.

(ii) A function $g: A^{n} \rightarrow B$ is a reconstruction of $f$, if $\operatorname{deck} f=\operatorname{deck} g$, or, equivalently, if there exists a bijection $\phi:\left(\begin{array}{c}{[n]} \\ 2\end{array}\right) \rightarrow\left(\begin{array}{c}{[n]} \\ 2\end{array}\right)$ such that $f_{I} \equiv g_{\phi(I)}$ for all $I \in\left(\begin{array}{c}{[n]} \\ 2\end{array}\right)$.

(iii) A function is reconstructible if it is equivalent to all of its reconstructions, or, equivalently, if it is equivalent to all functions with the same deck.

(iv) A parameter defined for all functions is reconstructible if it has the same value for all the reconstructions of any function.

(v) A class $\mathcal{C} \subseteq \mathcal{F}_{A B}$ of functions is recognizable if all reconstructions of the members of $\mathcal{C}$ are members of $\mathcal{C}$.

(vi) A class $\mathcal{C} \subseteq \mathcal{F}_{A B}$ of functions is weakly reconstructible if for every $f \in \mathcal{C}$, all reconstructions of $f$ that are members of $\mathcal{C}$ are equivalent to $f$.

(vii) A class $\mathcal{C} \subseteq \mathcal{F}_{A B}$ of functions is reconstructible if all members of $\mathcal{C}$ are reconstructible.

Note that if a class of functions is recognizable and weakly reconstructible, then it is reconstructible.

Using this terminology, we can now address the problem whether and to what extent functions of several arguments are reconstructible. Perhaps one of the simplest and most obvious questions to ask is the following.

Question 3.1. Let $A$ and $B$ be sets with at least two elements, and let $n$ be an integer greater than or equal to 2 . Is every function $f: A^{n} \rightarrow B$ reconstructible?

Remark 3.2. The answer to Question 3.1 is negative if $n$ is not sufficiently large. Namely, if $n \leqslant|A|$, then the set

$$
A_{\neq}^{n}:=\left\{\left(a_{1}, \ldots, a_{n}\right) \in A^{n}: a_{1}, a_{2}, \ldots, a_{n} \text { are pairwise distinct }\right\}
$$

is nonempty. In this case, if $f$ and $g$ are $n$-ary functions that coincide on $A^{n} \backslash A_{\neq}^{n}$, then $f_{I}=g_{I}$ for every $I \in\left(\begin{array}{c}{[n]} \\ 2\end{array}\right)$ but $f$ and $g$ need not be equivalent-consider, for example, any functions $f$ and $g$ that coincide on $A^{n} \backslash A_{\neq}^{n}$ such that $\left.f\right|_{A_{\neq}^{n}}$ is constant and $\left.g\right|_{A_{\neq}^{n}}$ is nonconstant. Therefore, no function $f: A^{n} \rightarrow B$ with $n \leqslant|A|$ is reconstructible. This also shows that functions with infinite domains are not reconstructible. Furthermore, Example 3.13 shows that the answer to Question 3.1 is negative if $n=|A|+1$. 


\subsection{Examples of reconstructible functions: constant functions}

Example 3.3. It is easy to verify that if $f: A^{n} \rightarrow B$ is a constant function and $n>|A|$, then $f$ is reconstructible. For, if $\alpha \in B$ and $f(\mathbf{a})=\alpha$ for all $\mathbf{a} \in A^{n}$, then for every $I \in\left(\begin{array}{c}{[n]} \\ 2\end{array}\right), f_{I}(\mathbf{b})=\alpha$ for all $\mathbf{b} \in A^{n-1}$. Assume that $g: A^{n} \rightarrow B$ is a reconstruction of $f$. Then there exists a bijection $\sigma:\left(\begin{array}{c}{[n]} \\ 2\end{array}\right) \rightarrow\left(\begin{array}{c}{[n]} \\ 2\end{array}\right)$ such that $f_{I} \equiv g_{\sigma(I)}$ for all $I \in\left(\begin{array}{c}{[n]} \\ 2\end{array}\right)$, and for each $I \in\left(\begin{array}{c}{[n]} \\ 2\end{array}\right)$, there exists a permutation $\rho_{I} \in \Sigma_{n-1}$ such that $f_{I}(\mathbf{b})=g_{\sigma(I)}\left(\mathbf{b} \rho_{I}\right)$ for all $\mathbf{b} \in A^{n-1}$. Let $\mathbf{a} \in A^{n}$. Since $n>|A|$, there exist $\mathbf{b} \in A^{n-1}$ and $I \in\left(\begin{array}{c}{[n]} \\ 2\end{array}\right)$ such that $\mathbf{a}=\mathbf{b} \delta_{I}$. Then $g(\mathbf{a})=g\left(\mathbf{b} \delta_{I}\right)=g_{I}(\mathbf{b})=f_{\sigma(I)}\left(\mathbf{b} \rho_{I}\right)=\alpha$. Thus $f=g$, and we conclude that $f$ is reconstructible.

\subsection{Further examples of reconstructible functions: functions determined by supp and oddsupp}

Following Berman and Kisielewicz [2], we define the maps supp: $\bigcup_{n \geqslant 1} A^{n} \rightarrow \mathcal{P}(A)$ and oddsupp: $\bigcup_{n \geqslant 1} A^{n} \rightarrow \mathcal{P}(A)$ by the rules

$$
\begin{aligned}
\operatorname{supp}\left(a_{1}, \ldots, a_{n}\right) & =\left\{a_{1}, \ldots, a_{n}\right\} \\
\text { oddsupp }\left(a_{1}, \ldots, a_{n}\right) & =\left\{a \in A:\left|\left\{i \in[n]: a_{i}=a\right\}\right| \text { is odd }\right\} .
\end{aligned}
$$

A function $f: A^{n} \rightarrow B$ is determined by supp, if there exists a map $f^{*}: \mathcal{P}(A) \rightarrow B$ such that $f=\left.f^{*} \circ \operatorname{supp}\right|_{A^{n}}$. Similarly, a function $f: A^{n} \rightarrow B$ is determined by oddsupp, if there exists a map $f^{*}: \mathcal{P}(A) \rightarrow B$ such that $f=f^{*}$ o oddsupp $\left.\right|_{A^{n}}$.

Remark 3.4. Functions determined by supp or oddsupp are totally symmetric. Hence, they depend on all of their arguments or on none of them.

Remark 3.5. For all $\mathbf{a} \in A^{n-1}$ and for all $I \in\left(\begin{array}{c}{[n]} \\ 2\end{array}\right)$, we have that $\operatorname{supp}(\mathbf{a})=\operatorname{supp}\left(\mathbf{a} \delta_{I}\right)$.

Remark 3.6. Let $f: A^{n} \rightarrow B$. It is easy to verify that if $f=\left.f^{*} \circ \operatorname{supp}\right|_{A^{n}}$ for some $f^{*}: \mathcal{P}(A) \rightarrow B$, then $f_{I}=f^{*}$ osupp $\left.\right|_{A^{n-1}}$ for all $I \in\left(\begin{array}{c}{[n]} \\ 2\end{array}\right)$. Also, if $f=f^{*}$ ooddsupp $\left.\right|_{A^{n}}$, then for all $I \in\left(\begin{array}{c}{[n]} \\ 2\end{array}\right)$, the $(\min I)$-th argument of $f_{I}$ is inessential and $f_{I} \equiv f^{*}$ o oddsupp $\left.\right|_{A^{n-2}}$.

For $n \geqslant 1$, denote

$$
\begin{aligned}
\mathcal{P}_{\leqslant n}(A) & :=\{S \subseteq A:|S| \leqslant n\}, \\
\mathcal{P}_{n}^{\prime}(A) & :=\{S \subseteq A:|S| \in\{n, n-2, n-4, \ldots\}\} .
\end{aligned}
$$

The range of $\left.\operatorname{supp}\right|_{A^{n}}$ equals $\mathcal{P}_{\leqslant n}(A)$. Thus, only the restriction of $f^{*}$ to $\mathcal{P}_{\leqslant n}(A)$ is relevant for the composition $\left.f^{*} \circ \operatorname{supp}\right|_{A^{n}}$, and $\left.f^{*} \circ \operatorname{supp}\right|_{A^{n}}=\left.g^{*} \circ \operatorname{supp}\right|_{A^{n}}$ if and only if $\left.f^{*}\right|_{\mathcal{P}_{\leqslant n}(A)}=\left.g^{*}\right|_{\mathcal{P}_{\leqslant n}(A)}$. Similarly, the range of oddsupp $\left.\right|_{A^{n}}$ equals $\mathcal{P}_{n}^{\prime}(A)$. Thus, only the restriction of $f^{*}$ to $\mathcal{P}_{n}^{\prime}(A)$ is relevant for the composition $f^{*}$ o oddsupp $\left.\right|_{A^{n}}$, and $f^{*} \circ$ oddsupp $\left.\right|_{A^{n}}=g^{*}$ o oddsupp $\left.\right|_{A^{n}}$ if and only if $\left.f^{*}\right|_{\mathcal{P}_{n}^{\prime}(A)}=\left.g^{*}\right|_{\mathcal{P}_{n}^{\prime}(A)}$.

Let us recall a useful result about functions determined by oddsupp from the paper by Willard [35]. 
Lemma 3.7 (Willard [35, Corollary 2.3]). Assume that $n>\max (|A|, 3)$ and $f: A^{n} \rightarrow B$ depends on all of its arguments. If no identification minor of $f$ depends on all of its arguments, then $f$ is determined by oddsupp.

The reconstructibility of functions determined by supp or oddsupp (of sufficiently large arity) now follows almost immediately.

Proposition 3.8. Let $f: A^{n} \rightarrow B$, and assume that $n>|A|$ and $f$ is determined by supp. Then the function $f$ is reconstructible.

Proof. Since functions determined by supp are totally symmetric, $f$ depends either on all of its arguments or on none of them. If $f$ has no essential arguments, then it is constant and hence it is reconstructible by Example 3.3. Assume thus that $f$ depends on all of its arguments. Then there exists a map $f^{*}: \mathcal{P}(A) \rightarrow B$ such that $f=\left.f^{*} \circ \operatorname{supp}\right|_{A^{n}}$ and $\left.f^{*}\right|_{\mathcal{P}_{\leqslant n}(A)}$ is not a constant function. By Remark 3.6, $f_{I}=\left.f^{*} \circ \operatorname{supp}\right|_{A^{n-1}}$ for all $I \in\left(\begin{array}{c}{[n]} \\ 2\end{array}\right)$. Since $n>|A|$, we have that $\mathcal{P}_{\leqslant n-1}(A)=\mathcal{P}(A)=\mathcal{P}_{\leqslant n}(A)$; hence $f_{I}$ depends on all of its $n-1$ arguments. Let $g: A^{n} \rightarrow B$ be a reconstruction of $f$. Then $g_{I} \equiv f^{*}$ osupp $\left.\right|_{A^{n-1}}$ for all $I \in\left(\begin{array}{c}{[n]} \\ 2\end{array}\right)$. Since $\left.f^{*} \circ \operatorname{supp}\right|_{A^{n-1}}$ is totally symmetric, we have in fact that $g_{I}=\left.f^{*} \circ \operatorname{supp}\right|_{A^{n-1}}$ for all $I \in\left(\begin{array}{c}{[n]} \\ 2\end{array}\right)$.

We claim that $g=f$. In order to prove this, let $\mathbf{a} \in A^{n}$. Since $n>|A|$, there exist $\mathbf{b} \in A^{n-1}$ and $I \in\left(\begin{array}{c}{[n]} \\ 2\end{array}\right)$ such that $\mathbf{a}=\mathbf{b} \delta_{I}$. Then

$$
g(\mathbf{a})=g\left(\mathbf{b} \delta_{I}\right)=g_{I}(\mathbf{b})=f^{*}(\operatorname{supp}(\mathbf{b}))=f^{*}\left(\operatorname{supp}\left(\mathbf{b} \delta_{I}\right)\right)=f^{*}(\operatorname{supp}(\mathbf{a}))=f(\mathbf{a}) .
$$

Thus $g=f$, and we conclude that $f$ is reconstructible.

Proposition 3.9. Let $f: A^{n} \rightarrow B$, and assume that $n>\max (|A|, 3)$ and $f$ is determined by oddsupp. Then the function $f$ is reconstructible.

Proof. Functions determined by oddsupp are totally symmetric, so we can assume, as in the proof of Proposition 3.8, that $f$ depends on all of its arguments. Then there exists a map $f^{*}: \mathcal{P}(A) \rightarrow B$ such that $f=f^{*}$ ooddsupp $\left.\right|_{A^{n}}$ and $\left.f^{*}\right|_{\mathcal{P}_{n}^{\prime}(A)}$ is not a constant function. By Remark 3.6, for all $I \in\left(\begin{array}{c}{[n]} \\ 2\end{array}\right), f_{I}$ has an inessential argument and $f_{I} \equiv f^{*}$ ooddsupp $\left.\right|_{A^{n-2}}$. Since $n>|A|$, it holds that $\mathcal{P}_{n-2}^{\prime}(A)=\mathcal{P}_{n}^{\prime}(A)$. Consequently, $\left.f^{*}\right|_{\mathcal{P}_{n-2}^{\prime}(A)}$ is not a constant function, so $f^{*}$ o oddsupp $\left.\right|_{A^{n-2}}$ depends on all of its arguments. We conclude that $f_{I}$ has exactly $n-2$ essential arguments, for any $I \in\left(\begin{array}{c}{[n]} \\ 2\end{array}\right)$.

Let $g: A^{n} \rightarrow B$ be a reconstruction of $f$. We claim that $g$ depends on all of its arguments. Suppose, on the contrary, that $g$ has an inessential argument, say, the $i$-th argument is inessential in $g$. Let $r$ be the number of essential arguments of $g$; it clearly holds that $0 \leqslant r \leqslant n-1$. If $r \neq n-2$, then for $j \in[n] \backslash\{i\}$, we have $g_{\{i, j\}} \equiv g$, and $g$ has an identification minor with $r$ essential arguments, contradicting deck $f=\operatorname{deck} g$. Otherwise $r=n-2$. Since $n \geqslant 4$, there are dictinct $p, q \in[n]$ such that the $p$-th and the $q$-th arguments are essential in $g$. Then $g_{\{p, q\}}$ depends on fewer than $n-2$ arguments, contradicting again $\operatorname{deck} f=\operatorname{deck} g$. We conclude that $g$ depends on all of its arguments, as claimed. 
Lemma 3.7 implies that $g$ is determined by oddsupp. Thus, $g=g^{*} \circ$ oddsupp $\left.\right|_{A^{n}}$ for some $g: \mathcal{P}(A) \rightarrow B$. By Remark 3.6, $g_{I} \equiv g^{*}$ ooddsupp $\left.\right|_{A^{n-2}}$ for all $I \in\left(\begin{array}{c}{[n]} \\ 2\end{array}\right)$. Then it holds that $f^{*} \circ$ oddsupp $\left.\right|_{A^{n-2}} \equiv g^{*} \circ$ oddsupp $\left.\right|_{A^{n-2}}$, because $\operatorname{deck} f=\operatorname{deck} g$. Since $\left.\operatorname{supp}\right|_{A^{n-1}}$ is totally symmetric, we have that $f^{*}$ o oddsupp $\left.\right|_{A^{n-2}}=g^{*}$ o oddsupp $\left.\right|_{A^{n-2}}$, and Remark 3.6 implies that $\left.f^{*}\right|_{\mathcal{P}_{n-2}^{\prime}(A)}=\left.g^{*}\right|_{\mathcal{P}_{n-2}^{\prime}(A)}$. As we have already observed, $\mathcal{P}_{n-2}^{\prime}(A)=\mathcal{P}_{n}^{\prime}(A)$, and it follows that $f=\left.f^{*}\right|_{\mathcal{P}_{n}^{\prime}(A)} \circ$ oddsupp $\left.\right|_{A^{n}}=\left.g^{*}\right|_{\mathcal{P}_{n}^{\prime}(A)} \circ$ oddsupp $\left.\right|_{A^{n}}=g$. We conclude that $f$ is reconstructible.

We are going to extend these results in Section 5, in which we prove that all totally symmetric functions of sufficiently large arity are reconstructible. Let us still note that it follows from Remark 3.2 that in Proposition 3.8 the lower bound $n>|A|$ is sharp, and in Proposition 3.9 the condition $n>|A|$ is necessary. The following example shows that the lower bound $n \geqslant 4$ is sharp for $|A|=2$ in Proposition 3.9.

Example 3.10. Let $A=\{0,1\}$, let $a, b, c, d \in B$ with $b \neq c$, and let $f, g, h: A^{3} \rightarrow B$ be given by the following table.

\begin{tabular}{|ccc|ccc|}
\hline$x$ & $y$ & $z$ & $f(x, y, z)$ & $g(x, y, z)$ & $h(x, y, z)$ \\
\hline 0 & 0 & 0 & $a$ & $a$ & $a$ \\
0 & 0 & 1 & $b$ & $c$ & $b$ \\
0 & 1 & 0 & $b$ & $c$ & $b$ \\
0 & 1 & 1 & $c$ & $b$ & $b$ \\
1 & 0 & 0 & $b$ & $c$ & $c$ \\
1 & 0 & 1 & $c$ & $b$ & $c$ \\
1 & 1 & 0 & $c$ & $b$ & $c$ \\
1 & 1 & 1 & $d$ & $d$ & $d$ \\
\hline
\end{tabular}

Functions $f$ and $g$ are totally symmetric, but $h$ is not totally symmetric and not even 2 -set-transitive (see Section 4). These functions are pairwise nonequivalent. Furthermore, if $a=c$ and $b=d$, then $f$ is determined by oddsupp (or if $a=b$ and $c=d$, then $g$ is determined by oddsupp).

It is not difficult to verify that for all $I \in\left(\begin{array}{c}{[n]} \\ 2\end{array}\right)$, each one of $f_{I}, g_{I}$, and $h_{I}$ is equivalent to the function $(0,0) \mapsto a,(0,1) \mapsto b,(1,0) \mapsto c,(1,1) \mapsto d$. Hence $f, g$, and $h$ are reconstructions of each other.

\subsection{Examples of nonreconstructible functions}

We present here a scheme for producing functions of arity $|A|+1$ with a predetermined deck of a special form. With a suitable choice of parameters, nonequivalent functions with the same deck will arise.

Definition 3.11. Assume that $n=k+1$, and let $A$ be a set such that $|A|=k$. Let $g^{*}: \mathcal{P}(A) \rightarrow B$ and let $g: A^{k} \rightarrow B, g=\left.g^{*} \circ \operatorname{supp}\right|_{A^{k}}$. Let $G:=\left(g^{I}\right)_{I \in\left(\begin{array}{c}{[n]} \\ 2\end{array}\right)}$ be a family of functions $g^{I}: A^{k} \rightarrow B$ satisfying $g^{I}(\mathbf{a})=g(\mathbf{a})$ whenever $\operatorname{supp}(\mathbf{a}) \neq A$, and let $P:=$ 
$\left(\rho_{I}\right)_{I \in\left(\begin{array}{c}{[n]} \\ 2\end{array}\right)}$ be a family of permutations from $\Sigma_{k}$. Let $\phi:\left(\begin{array}{c}{[n]} \\ 2\end{array}\right) \rightarrow\left(\begin{array}{c}{[n]} \\ 2\end{array}\right)$ be a bijection. Define $f_{G, P, \phi}: A^{n} \rightarrow B$ by the rule $f_{G, P, \phi}(\mathbf{b})=g^{\phi(I)}\left(\mathbf{a} \rho_{I}\right)$ if $\mathbf{b}=\mathbf{a} \delta_{I}$. This function is well defined, because if $\operatorname{supp}(\mathbf{b})=A$, then there is a unique $\mathbf{a} \in A^{k}$ and a unique $I \in\left(\begin{array}{c}{[n]} \\ 2\end{array}\right)$ such that $\mathbf{b}=\mathbf{a} \delta_{I}$; and if $\operatorname{supp}(\mathbf{b}) \neq A$, then for every $\mathbf{a} \in A^{k}$ and $I \in\left(\begin{array}{c}{[n]} \\ 2\end{array}\right)$ satisfying $\mathbf{b}=\mathbf{a} \delta_{I}$, we have $\operatorname{supp}(\mathbf{a})=\operatorname{supp}(\mathbf{b}) \neq A$ and $g^{\phi(I)}\left(\mathbf{a} \rho_{I}\right)=g^{*}\left(\operatorname{supp}\left(\mathbf{a} \rho_{I}\right)\right)=g^{*}(\operatorname{supp}(\mathbf{b}))$.

Lemma 3.12. For any family $G:=\left(g^{I}\right)_{I \in\left(\begin{array}{c}{[n]} \\ 2\end{array}\right)}$ of functions, any family $P:=\left(\rho_{I}\right)_{I \in\left(\begin{array}{c}{[n]} \\ 2\end{array}\right)}$ of permutations, and any bijection $\phi:\left(\begin{array}{c}{[n]} \\ 2\end{array}\right) \rightarrow\left(\begin{array}{c}{[n]} \\ 2\end{array}\right)$ as in Definition 3.11, it holds that $\left(f_{G, P, \phi}\right)_{I} \equiv g^{\phi(I)}$ for every $I \in\left(\begin{array}{c}{[n]} \\ 2\end{array}\right)$. Consequently, deck $f_{G, P, \phi}=\left\langle g^{I} / \equiv: I \in\left(\begin{array}{c}{[n]} \\ 2\end{array}\right)\right\rangle$.

Proof. It follows directly from the definitions that, for all $\mathbf{a} \in A^{k}$,

$$
\left(f_{G, P, \phi}\right)_{I}(\mathbf{a})=f_{G, P, \phi}\left(\mathbf{a} \delta_{I}\right)=g^{\phi(I)}\left(\mathbf{a} \rho_{I}\right),
$$

that is, $\left(f_{G, P, \phi}\right)_{I} \equiv g^{\phi(I)}$. The claim about $\operatorname{deck} f_{G, P, \phi}$ follows immediately.

Thus, for a fixed family $G$ of functions and for families $P$ and $P^{\prime}$ of permutations and for bijections $\phi$ and $\phi^{\prime}$, the functions $f_{G, P, \phi}$ and $f_{G, P^{\prime}, \phi^{\prime}}$ are reconstructions of each other but they are not necessarily equivalent. As the following example illustrates, it is indeed possible that $f_{G, P, \phi} \not \equiv f_{G, P^{\prime}, \phi^{\prime}}$ for a suitable choice of $G, P, P^{\prime}, \phi$, and $\phi^{\prime}$. Thus, the answer to Question 3.1 is negative if $n=|A|+1$.

Example 3.13. Let $n=k+1$, and let $A$ and $B$ be sets such that $|A|=k$ and $A \subseteq B$, and let $\beta \in B$. Define the functions $f, f^{\prime}: A^{n} \rightarrow B$ by the rules

$$
\begin{aligned}
f(\mathbf{a}) & = \begin{cases}b, & \text { if } \operatorname{supp}(\mathbf{a})=A \text { and } b \text { is an element of } A \text { that occurs twice in } \mathbf{a}, \\
\beta, & \text { otherwise }\end{cases} \\
f^{\prime}(\mathbf{a}) & = \begin{cases}a_{1}, & \text { if } \operatorname{supp}(\mathbf{a})=A, \\
\beta, & \text { otherwise. }\end{cases}
\end{aligned}
$$

Note that $f$ is well defined, because if $\operatorname{supp}(\mathbf{a})=A$, then every element of $A$ occurs in a, and since $n=|A|+1$, there exists a unique element of $A$ that occurs twice in a. It is easy to verify that $f \not \equiv f^{\prime}$. To see this, note that if $c$ and $d$ are distinct elements of $A$, then, on the one hand, there exists a tuple $\mathbf{a} \in A^{n}$ such that $\operatorname{supp}(\mathbf{a})=A$, a has two occurrences of $c$ and $f^{\prime}(\mathbf{a})=d$, but, on the other hand, for every tuple $\mathbf{b} \in A^{n}$ such that $\operatorname{supp}(\mathbf{b})=A$ and $\mathbf{b}$ has two occurrences of $c$, it holds that $f(\mathbf{b})=c$.

Let us now present parameters $G, P, P^{\prime}, \phi, \phi^{\prime}$ that give rise to $f$ and $f^{\prime}$ as $f_{G, P, \phi}$ and $f_{G, P^{\prime}, \phi^{\prime}}$, respectively, as in Definition 3.11. Let $g^{*}: \mathcal{P}(A) \rightarrow B$ be the constant map that sends every $S \in \mathcal{P}(A)$ to $\beta$, and let $g:=\left.g^{*} \circ \operatorname{supp}\right|_{A^{k}}$. For every $I \in\left(\begin{array}{c}{[n]} \\ 2\end{array}\right)$, let $g^{I}: A^{k} \rightarrow B$ be the function

$$
g^{I}(\mathbf{a})= \begin{cases}a_{1}, & \text { if } \operatorname{supp}(\mathbf{a})=A \\ \beta, & \text { otherwise }\end{cases}
$$


We clearly have that $g^{I}$ satisfies the condition that $g^{I}(\mathbf{a})=g(\mathbf{a})$ whenever $\operatorname{supp}(\mathbf{a}) \neq A$. For each $I \in\left(\begin{array}{c}{[n]} \\ 2\end{array}\right)$, let $\rho_{I}$ be the identity permutation if $\min I=1$, and let $\rho_{I}$ be the transposition of 1 and $\min I$ if $\min I \neq 1$; and let $\rho_{I}^{\prime}$ be the identity permutation. Let both $\phi$ and $\phi^{\prime}$ be equal to the identity map on $\left(\begin{array}{c}{[n]} \\ 2\end{array}\right)$. Denote $G:=\left(g^{I}\right)_{I \in\left(\begin{array}{c}{[n]} \\ 2\end{array}\right)}, P:=\left(\rho_{I}\right)_{I \in\left(\begin{array}{c}{[n]} \\ 2\end{array}\right)}$, and $P^{\prime}:=\left(\rho_{I}^{\prime}\right)_{I \in\left(\begin{array}{c}{[n]} \\ 2\end{array}\right)}$. We leave it to the reader to verify that indeed $f=f_{G, P, \phi}$ and $f^{\prime}=f_{G, P^{\prime}, \phi^{\prime}}$. Thus, by Lemma 3.12, we have that $f_{I} \equiv g^{I} \equiv f_{I}^{\prime}$ for all $I \in\left(\begin{array}{c}{[n]} \\ 2\end{array}\right)$, so $\operatorname{deck} f=\operatorname{deck} f^{\prime}$.

We conclude that $f$ and $f^{\prime}$ are not reconstructible. Note also that the function $f$ is totally symmetric, but $f^{\prime}$ is not totally symmetric and not even 2-set-transitive (see Section 4).

\subsection{Examples of reconstructible parameters}

Example 3.14. Let $f: A^{n} \rightarrow B$. The diagonal of $f$ is the map $\Delta_{f}: A \rightarrow B$ given by $\Delta_{f}(a)=f(a, a, \ldots, a)$ for all $a \in A$. It is easy to verify that the diagonal of every minor of $f$ equals $\Delta_{f}$. It follows that the diagonal is a reconstructible parameter of functions.

\subsection{Examples of recognizable classes of functions}

Let $\mathcal{C} \subseteq \mathcal{F}_{A B}$ be a class of functions, and let $d$ and $r$ be positive integers. Let $S \subseteq\left(A^{r}\right)^{d}$ and $T \subseteq B^{r}$. The couple $(S, T)$ is called a d-dimensional nonmembership witness scheme for $\mathcal{C}$, if it holds for every $n \geqslant 1$ and $f \in \mathcal{F}_{A B}^{(n)}$ that $f \notin \mathcal{C}$ if and only if there exists elements $a_{i j} \in A(1 \leqslant i \leqslant r, 1 \leqslant j \leqslant n)$ and a map $\rho:[d] \rightarrow[n]$ such that $a_{1 j}=a_{2 j}=\cdots=a_{r j}$ for every $j \in[n] \backslash \operatorname{Im} \rho$ and

$$
\begin{gathered}
\left(\left(a_{1 \rho(1)}, a_{2 \rho(1)}, \ldots, a_{r \rho(1)}\right), \ldots,\left(a_{1 \rho(d)}, a_{2 \rho(d)}, \ldots, a_{r \rho(d)}\right)\right) \in S \\
\left(f\left(a_{11}, a_{12}, \ldots, a_{1 n}\right), \ldots, f\left(a_{r 1}, a_{r 2}, \ldots, a_{r n}\right)\right) \in T .
\end{gathered}
$$

Proposition 3.15. Assume that $\mathcal{C} \subseteq \mathcal{F}_{A B}$ is a class of functions that is closed under formation of minors and there exists a d-dimensional nonmembership witness scheme $(S, T)$ for $\mathcal{C}$. Then the class $\mathcal{D}:=\bigcup_{n \geqslant d+|A|+1} \mathcal{C}^{(n)}$ is recognizable.

Proof. Let $f: A^{n} \rightarrow B$ with $n \geqslant d+|A|+1$. We claim that $f_{I} \in \mathcal{C}$ for every $I \in\left(\begin{array}{c}{[n]} \\ 2\end{array}\right)$ if and only if $f \in \mathcal{C}$. The sufficiency is clear, because $\mathcal{C}$ is closed under formation of minors. For necessity, assume that $f \notin \mathcal{C}$. Then there exist a positive integer $r$ and elements $a_{i j} \in A(1 \leqslant i \leqslant r, 1 \leqslant j \leqslant n)$ such that $a_{1 j}=a_{2 j}=\cdots=a_{r j}$ for every $j \in[n] \backslash \operatorname{Im} \rho$ and

$$
\begin{gathered}
\left(\left(a_{1 \rho(1)}, a_{2 \rho(1)}, \ldots, a_{r \rho(1)}\right), \ldots,\left(a_{1 \rho(d)}, a_{2 \rho(d)}, \ldots, a_{r \rho(d)}\right)\right) \in S \\
\left(f\left(a_{11}, a_{12}, \ldots, a_{1 n}\right), \ldots, f\left(a_{r 1}, a_{r 2}, \ldots, a_{r n}\right)\right) \in T .
\end{gathered}
$$

Since $n-d \geqslant|A|+1$, there exist indices $p, q \in[n] \backslash \operatorname{Im} \rho$ such that $a_{1 p}=a_{1 q}$. Set $J:=\{p, q\}$, and let $b_{i j} \in A(1 \leqslant i \leqslant r, 1 \leqslant j \leqslant n-1)$ be the unique elements such that 
$a_{i j}=b_{i, \delta_{J}(j)}$ for all $1 \leqslant i \leqslant r, 1 \leqslant j \leqslant n$, and let $\tau=\delta_{J} \circ \rho$. Then $b_{i, \tau(\ell)}=b_{i, \delta_{J}(\rho(\ell))}=a_{i, \rho(\ell)}$, and it is easy to verify that $b_{1 j}=b_{2 j}=\cdots=b_{r j}$ for every $j \in[n-1] \backslash \operatorname{Im} \tau$ and

$$
\begin{gathered}
\left(\left(b_{1 \tau(1)}, b_{2 \tau(1)}, \ldots, b_{r \tau(1)}\right), \ldots,\left(b_{1 \tau(d)}, b_{2 \tau(d)}, \ldots, b_{r \tau(d)}\right)\right) \in S, \\
\left(f_{J}\left(b_{11}, b_{12}, \ldots, b_{1, n-1}\right), \ldots, f_{J}\left(b_{r 1}, b_{r 2}, \ldots, b_{r, n-1}\right)\right) \in T .
\end{gathered}
$$

Thus, $f_{J} \notin \mathcal{C}$, because $(S, T)$ is a nonmembership witness scheme for $\mathcal{C}$.

This implies that every reconstruction of every member of $\mathcal{D}$ is again a member of $\mathcal{D}$, that is, $\mathcal{D}$ is recognizable.

Example 3.16. Let $\left(A ; \leqslant_{A}\right)$ and $\left(B ; \leqslant_{B}\right)$ be partially ordered sets. A function $f: A^{n} \rightarrow$ $B$ is order-preserving if for all $\mathbf{a}, \mathbf{b} \in A^{n}$, the condition $\mathbf{a} \leqslant{ }_{A} \mathbf{b}$ (i.e., $a_{i} \leqslant{ }_{A} b_{i}$ for all $i \in[n]$ ) implies $f(\mathbf{a}) \leqslant_{B} f(\mathbf{b})$. We claim that $\left(\leqslant_{A}, B^{2} \backslash \leqslant_{B}\right)$ is a 1-dimensional nonmembership witness scheme for the class of order-preserving functions from $A$ to $B$. Consequently, by Proposition 3.15, the class of order-preserving functions from $A$ to $B$ of arity at least $|A|+2$ is recognizable.

In order to prove the claim, observe first that if $f: A^{n} \rightarrow B$ is order-preserving, then for all $a_{i j}(1 \leqslant i \leqslant 2,1 \leqslant j \leqslant n)$ and for every $\rho:[1] \rightarrow[n]$ such that $a_{1 j}=a_{2 j}$ for all $j \in[n] \backslash \operatorname{Im} \rho$ and $a_{1 \rho(1)} \leqslant_{A} a_{2 \rho(2)}$, we have that $\left(a_{11}, \ldots, a_{1 n}\right) \leqslant_{A}\left(a_{2 n}, \ldots, a_{2 n}\right)$; hence $f\left(a_{11}, \ldots, a_{1 n}\right) \leqslant_{B} f\left(a_{21}, \ldots, a_{2 n}\right)$.

If $f: A^{n} \rightarrow B$ is not order-preserving, then there exist tuples $\mathbf{a}, \mathbf{b} \in A^{n}$ such that $\mathbf{a} \leqslant{ }_{A} \mathbf{b}$ and $f(\mathbf{a}) k_{B} f(\mathbf{b})$. Consider the sequence

$$
\begin{aligned}
\mathbf{c}_{1} & :=\left(a_{1}, a_{2}, a_{3}, \ldots, a_{n}\right)=\mathbf{a}, \\
\mathbf{c}_{2} & :=\left(b_{1}, a_{2}, a_{3}, \ldots, a_{n}\right), \\
\mathbf{c}_{3} & :=\left(b_{1}, b_{2}, a_{3}, \ldots, a_{n}\right), \\
& \vdots \\
\mathbf{c}_{n} & :=\left(b_{1}, \ldots, b_{n-1}, a_{n}\right), \\
\mathbf{c}_{n+1} & :=\left(b_{1}, \ldots, b_{n-1}, b_{n}\right)=\mathbf{b} .
\end{aligned}
$$

It holds that $\mathbf{c}_{\ell} \leqslant{ }_{A} \mathbf{c}_{\ell+1}$ for all $\ell \in[n]$. There exists an index $s \in[n]$ such that $f\left(\mathbf{c}_{s}\right) \mathbb{B}_{B}$ $f\left(\mathbf{c}_{s+1}\right)$ (otherwise we would have $f(\mathbf{a}) \leqslant_{B} f(\mathbf{b})$ by the transitivity of $\leqslant_{B}$, a contradiction). Choosing $a_{i j}:=\mathbf{c}_{s-i+1}(j)(1 \leqslant i \leqslant 2,1 \leqslant j \leqslant n)$ and $\rho:[1] \rightarrow[n], 1 \mapsto s$, the desired conditions for a nonmembership witness scheme are satisfied.

\section{On 2-set-transitivity and unique identification mi- nors}

In this section, we will prove an auxiliary result (Proposition 4.3) that is needed for the investigation of reconstructibility of totally symmetric functions in Section 5. The proposition will be formulated a bit more generally than is needed for our current application, and it asserts that the identification minors of any 2-set-transitive function are all equivalent to each other. 
We denote the symmetric group on $[n]$ by $\Sigma_{n}$. A function $f: A^{n} \rightarrow B$ is invariant under a permutation $\sigma \in \Sigma_{n}$, if for all $\mathbf{a} \in A^{n}$ it holds that $f(\mathbf{a})=f(\mathbf{a} \sigma)$. Let $\operatorname{Inv} f$ denote the set of permutations of $[n]$ under which $f$ is invariant. Clearly every function is invariant under the identity permutation. If $f$ is invariant under $\sigma$ and $\sigma^{\prime}$, then $f$ is also invariant under $\sigma^{-1}$ and $\sigma \sigma^{\prime}$. Hence Inv $f$ constitutes a subgroup of $\Sigma_{n}$, and it is called the invariance group of $f$. Note that $\operatorname{Inv} f=\Sigma_{n}$ if and only if $f$ is totally symmetric.

A permutation group $G \leqslant \Sigma_{n}$ is called 2-set-transitive if for all $a_{1}, a_{2}, b_{1}, b_{2} \in[n]$ such that $a_{1} \neq a_{2}$ and $b_{1} \neq b_{2}$, there exists a permutation $\sigma \in G$ such that $\left\{\sigma\left(a_{1}\right), \sigma\left(a_{2}\right)\right\}=$ $\left\{b_{1}, b_{2}\right\}$. A function $f: A^{n} \rightarrow B$ is 2-set-transitive if the invariance group of $f$ is 2 -settransitive. It is clear that totally symmetric functions are 2-set-transitive.

For $I \in\left(\begin{array}{c}{[n]} \\ 2\end{array}\right)$ and $\sigma \in \Sigma_{n}$, we write $I \sigma:=\{\sigma(i): i \in I\}$.

Lemma 4.1. Let $\sigma \in \Sigma_{n}$ and $I \in\left(\begin{array}{c}{[n]} \\ 2\end{array}\right)$. Then there exists a permutation $\hat{\sigma} \in \Sigma_{n-1}$ that satisfies $\hat{\sigma} \circ \delta_{I \sigma^{-1}}=\delta_{I} \circ \sigma$ and $\hat{\sigma}\left(\min I \sigma^{-1}\right)=\min I$.

Proof. Observe first that for any $J \in \Sigma_{n}$, the restriction of $\delta_{J}$ to the set $[n] \backslash\{\max J\}$ is a bijection. Define the map $\beta_{J}:[n-1] \rightarrow[n]$ as $\beta_{J}(\ell)=\left(\left.\left(\delta_{J}\right)\right|_{[n] \backslash\{\max J\}}\right)^{-1}(\ell)$ for all $\ell \in[n-1]$. In other words, $\beta_{J}$ is obtained from $\left(\left.\left(\delta_{J}\right)\right|_{[n] \backslash\{\max J\}}\right)^{-1}$ by extending the codomain; both maps are given by the rule $\ell \mapsto \ell$ for $1 \leqslant \ell<\max J$ and $\ell \mapsto \ell+1$ for $\max J \leqslant \ell \leqslant n-1$. Then $\beta_{J} \circ \delta_{J}:[n] \rightarrow[n]$ is the map $\ell \mapsto \ell$ for $\ell \neq \max J$ and $\max J \mapsto \min J$.

Let $\sigma \in \Sigma_{n}$ and $I \in\left(\begin{array}{c}{[n]} \\ 2\end{array}\right)$. We have that $\left(\delta_{I} \circ \sigma\right)\left(\sigma^{-1}(\min I)\right)=\left(\delta_{I} \circ \sigma\right)\left(\sigma^{-1}(\max I)\right)=$ $\min I$. Based on the above observations, it is easy to see that $\hat{\sigma}:=\delta_{I} \circ \sigma \circ \beta_{I \sigma^{-1}}$ is a permutation of $[n-1]$. Furthermore, $\hat{\sigma} \circ \delta_{I \sigma^{-1}}=\delta_{I} \circ \sigma \circ \beta_{I \sigma^{-1}} \circ \delta_{I \sigma^{-1}}=\delta_{I} \circ \sigma$.

Finally, observe that $\hat{\sigma}\left(\min I \sigma^{-1}\right)=\delta\left(\sigma\left(\beta_{I \sigma^{-1}}\left(\min I \sigma^{-1}\right)\right)\right)=\delta_{I}\left(\sigma\left(\min I \sigma^{-1}\right)\right)$. Since $\sigma\left(\min I \sigma^{-1}\right) \in I$ and $\delta_{I}$ maps both elements of $I$ to $\min I$, we have that $\delta_{I}\left(\sigma\left(\min I \sigma^{-1}\right)\right)=$ $\min I$.

Lemma 4.2. Assume that $f: A^{n} \rightarrow B$ is invariant under a permutation $\sigma \in \Sigma_{n}$. Then $f_{I} \equiv f_{I \sigma}$ for all $I \in\left(\begin{array}{c}n \\ 2\end{array}\right)$.

Proof. Let $\sigma \in \operatorname{Inv} f$ and $I \in\left(\begin{array}{c}{[n]} \\ 2\end{array}\right)$. Let $\hat{\sigma}$ be the permutation of $[n-1]$ given by Lemma 4.1. We have that for all $\mathbf{a} \in A^{n-1}$,

$$
f_{I}(\mathbf{a})=f\left(\mathbf{a} \delta_{I}\right)=f\left(\mathbf{a} \delta_{I} \sigma\right)=f\left(\mathbf{a} \hat{\sigma} \delta_{I \sigma^{-1}}\right)=f_{I \sigma^{-1}}(\mathbf{a} \hat{\sigma}) .
$$

The first and the last equalities hold by the definition of identification minor. The second equality holds because $f$ is invariant under $\sigma$. The third equality holds by Lemma 4.1 . We conclude that $f_{I} \equiv f_{I \sigma^{-1}}$, whence the claim follows.

Proposition 4.3. If $f: A^{n} \rightarrow B$ is 2-set-transitive, then $f_{I} \equiv f_{J}$ for all $I, J \in\left(\begin{array}{c}{[n]} \\ 2\end{array}\right)$.

Proof. Let $I, J \in\left(\begin{array}{c}{[n]} \\ 2\end{array}\right)$. By the 2-set-transitivity of $f$, there exists a permutation $\sigma \in \operatorname{Inv} f$ such that $I \sigma=J$. Lemma 4.2 implies that $f_{I} \equiv f_{J}$. 
These observations naturally lead to the following definition and problem that are of interest on their own right, even outside of the context of the reconstruction problem for functions. We say that a function $f: A^{n} \rightarrow B$ has a unique identification minor if $f_{I} \equiv f_{J}$ for all $I, J \in\left(\begin{array}{c}{[n]} \\ 2\end{array}\right)$.

Problem 4.4. Characterize the functions that have a unique identification minor.

To the best of the author's knowledge, this is an open problem. By Proposition 4.3, the 2-set-transitive functions have a unique identification minor. There exist functions with a unique identification minor that are not 2-set-transitive, e.g., the function $h$ of Example 3.10 and the function $f^{\prime}$ of Example 3.13.

Problem 4.4 was raised also by Bouaziz, Couceiro and Pouzet (see Problem 2(ii) in [6]). They considered the closely related problem of characterizing join-irreducible functions, i.e., functions with a unique lower cover in the minor partial order. Having a unique identification minor is a stronger condition than join-irreducibility. Indeed, if a function $f$ has a unique identification minor, then this unique minor is obviously the unique lower cover of $f$. The following example illustrates that these two conditions are not equivalent.

Example 4.5. Let $(A ; \wedge, \vee)$ be a lattice. Define the function $f: A^{4} \rightarrow A$ by the rule

$$
f\left(x_{1}, x_{2}, x_{3}, x_{4}\right)=\left(x_{1} \wedge x_{2}\right) \vee\left(x_{1} \wedge x_{3}\right) \vee\left(x_{1} \wedge x_{4}\right) \vee\left(x_{2} \wedge x_{3} \wedge x_{4}\right)
$$

It is easy to verify that the identification minors of $f$ are

$$
f_{\{1,2\}}=f_{\{1,3\}}=f_{\{1,4\}}=\operatorname{pr}_{1}^{(3)}, \quad f_{\{2,3\}}=f_{\{2,4\}}=f_{\{3,4\}}=\mu,
$$

where $\mu: A^{3} \rightarrow A$ is given by

$$
\mu\left(x_{1}, x_{2}, x_{3}\right)=\left(x_{1} \wedge x_{2}\right) \vee\left(x_{1} \wedge x_{3}\right) \vee\left(x_{2} \wedge x_{3}\right) .
$$

Note also that $\mu_{I}$ is a projection for all $I \in\left(\begin{array}{c}{[3]} \\ 2\end{array}\right)$. This shows that $f$ has a unique lower cover in the minor partial order, i.e., $f$ is join-irreducible. It is obvious that $f$ does not have a unique identification minor.

In [6], Bouaziz, Couceiro and Pouzet represent each Boolean function $f:\{0,1\}^{n} \rightarrow$ $\{0,1\}$ by a hypergraph on $[n]$ whose edges correspond to the monomials of the unique multilinear polynomial over the two-element field representing $f$ (further details are provided in Section 6), and they describe the join-irreducible functions among those Boolean functions whose representation is a Steiner system or a graph. A noteworthy result (Theorem 21 in [6]) is that for the Boolean functions whose hypergraph representation is a Steiner system, join-irreducibility is equivalent to having a unique identification minor, and both conditions are equivalent to -2-monomorphicity of the Steiner system. 


\section{Totally symmetric functions are reconstructible}

We now focus on totally symmetric functions. Our main result, Theorem 5.1, asserts that totally symmetric functions $f: A^{n} \rightarrow B$ are reconstructible, provided $n \geqslant|A|+2$. This generalizes Propositions 3.8 and 3.9 except when $n=|A|+1$. We also show that totally symmetric functions $f: A^{n} \rightarrow B$ are weakly reconstructible if $n>\max (|A|, 3)$ (Proposition 5.2). The lower bound of Theorem 5.1 is sharp: in Example 3.13 we have already seen totally symmetric functions of arity $n=|A|+1$ that are not reconstructible. Example 3.10 shows that the lower bound of Proposition 5.2 is sharp when $|A|=2$.

Let ms: $\bigcup_{n \geqslant 1} A^{n} \rightarrow \mathcal{M}(A)$ be the map that sends each tuple to the multiset of its entries, i.e., $\operatorname{ms}\left(a_{1}, \ldots, a_{n}\right)=\left\langle a_{1}, \ldots, a_{n}\right\rangle$ for every $\left(a_{1}, \ldots, a_{n}\right) \in A^{n}(n \geqslant 1)$. It is easy to verify that a function $f: A^{n} \rightarrow B$ is totally symmetric if and only if $f=\left.f^{*} \circ \mathrm{ms}\right|_{A^{n}}$ for some $f^{*}: \mathcal{M}_{n}(A) \rightarrow B$.

Before stating the result, let us introduce a notational device that will be used many times in the sequel. We write expressions such as

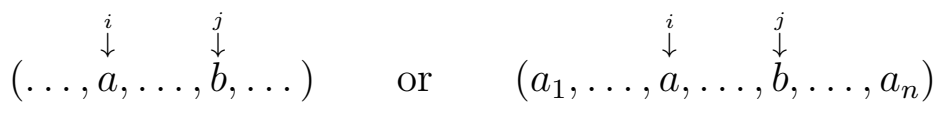

to denote an $n$-tuple whose $i$-th component is $a$ and the $j$-th component is $b$. The remaining components are irrelevant to the argument at hand and they are clear from the context. The indices $i$ and $j$ are always distinct and they may be equal to 1 or $n$, but it does not necessarily hold that $i<j$; however, if it is known that $i<j$, then we usually write the $i$-th component to the left of the $j$-th one. Also, whenever possible, we write components indexed by $i$ and $i+1$ next to each other, and we write components indexed by 1 or $n$ at the beginning and at the end of the tuple, respectively, as in the following:

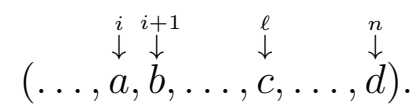

Theorem 5.1. Assume that $n \geqslant k+2$ and $|A|=k$. If $f: A^{n} \rightarrow B$ is totally symmetric, then $f$ is reconstructible.

Proof. Since $f$ is totally symmetric, there exists a map $f^{*}: \mathcal{M}_{n}(A) \rightarrow B$ such that $f=$ $\left.f^{*} \circ \mathrm{ms}\right|_{A^{n}}$. Let $h: A^{n-1} \rightarrow B$ be the function given by the rule $h(\mathbf{b})=f^{*}\left(\operatorname{ms}(\mathbf{b}) \uplus\left\langle b_{1}\right\rangle\right)$ for all $\mathbf{b} \in A^{n-1}$. Then for every $I \in\left(\begin{array}{c}{[n]} \\ 2\end{array}\right)$, it holds that $f_{I} \equiv h$. This innocent-looking fact will be crucial for the proof and justifies the formalism that we are going to build. Let $g: A^{n} \rightarrow B$ be a reconstruction of $f$. Then for every $I \in\left(\begin{array}{c}{[n]} \\ 2\end{array}\right)$, it holds that $g_{I} \equiv h$ and hence there exists a permutation $\rho_{I} \in \Sigma_{n-1}$ such that $g_{I}(\mathbf{b})=h\left(\mathbf{b} \rho_{I}\right)$ for all $\mathbf{b} \in A^{n-1}$. Let $q_{I}:=\min \delta_{I}^{-1}\left(\rho_{I}(1)\right)$.

Let $\mathbf{a} \in A^{n}$. Since $n \geqslant k+2$, there exist $I \in\left(\begin{array}{c}{[n]} \\ 2\end{array}\right)$ and $\mathbf{b} \in A^{n-1}$ such that $\mathbf{a}=\mathbf{b} \delta_{I}$. It holds that $g(\mathbf{a})=g\left(\mathbf{b} \delta_{I}\right)=g_{I}(\mathbf{b})=h\left(\mathbf{b} \rho_{I}\right)=f^{*}\left(\operatorname{ms}\left(\mathbf{b} \rho_{I}\right) \uplus\left\langle b_{\rho_{I}(1)}\right\rangle\right)$. Since $a_{i}=b_{\delta_{I}(i)}$ for every $i \in[n]$ and $\delta_{I}\left(q_{I}\right)=\rho_{I}(1)$, we have $b_{\rho_{I}(1)}=a_{q_{I}}$. Therefore, for any $I \in\left(\begin{array}{c}{[n]} \\ 2\end{array}\right)$ and for every $\mathbf{a} \in A^{n}$ such that $a_{\min I}=a_{\max I}$, it holds that

$$
g(\mathbf{a})=f^{*}\left(\operatorname{ms}(\mathbf{a}) \backslash\left\langle a_{\max I}\right\rangle \uplus\left\langle a_{q_{I}}\right\rangle\right) .
$$


In the sequel, we write " $\frac{(2)}{J}$ " for some $J \in\left(\begin{array}{c}{[n]} \\ 2\end{array}\right)$ to indicate that the equality in question holds by Equation (2) with $I=J$.

Claim 5.1.1. If there exist $I, J \in\left(\begin{array}{c}{[n]} \\ 2\end{array}\right)$ such that $I \cap J=\emptyset$ and $q_{I} \notin I$ and $q_{J} \notin J$, then $f^{*}(S)=f^{*}(T)$ for all $S, T \in \mathcal{M}_{n}(A)$ such that $\operatorname{set}(S)=\operatorname{set}(T)$.

Proof of Claim 5.1.1. Let $I=\{i, j\}, J=\{p, q\}$, and assume that $I \cap J=\emptyset$ and $q_{I} \notin I$ and $q_{J} \notin J$. We split the analysis into several cases.

Case 1: $q_{I}, q_{J} \notin I \cup J, q_{I} \neq q_{J}$. In this case $n \geqslant 6$. We have for any $\alpha, \beta, \gamma, \delta \in A$ and for any $\mathbf{u} \in A^{n-6}$ that

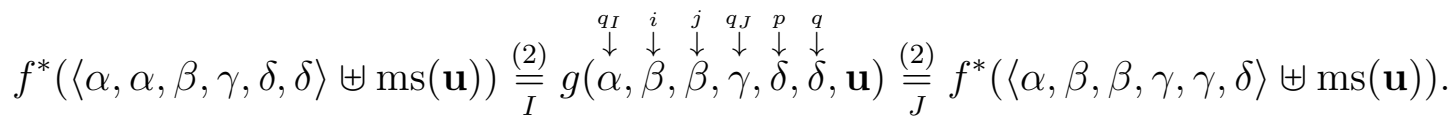

Let $K=\left\{q_{I}, q_{J}\right\}$. If $q_{K} \in K$, then for any $\alpha, \beta, \gamma, \delta \in A$ and for any $\mathbf{u} \in A^{n-6}$,

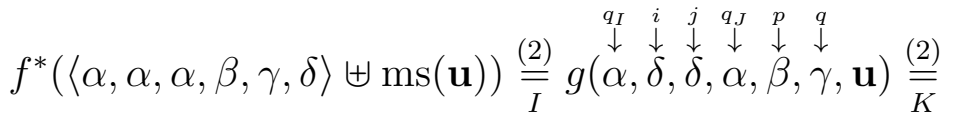

$$
\begin{aligned}
& f^{*}(\langle\alpha, \alpha, \beta, \gamma, \delta, \delta\rangle \uplus \operatorname{ms}(\mathbf{u}))=f^{*}(\langle\alpha, \beta, \beta, \gamma, \gamma, \delta\rangle \uplus \operatorname{ms}(\mathbf{u})) .
\end{aligned}
$$

If $q_{K} \notin K \cup I$, then for any $\alpha, \beta, \gamma \in A$ and for any $\mathbf{u} \in A^{n-5}$,

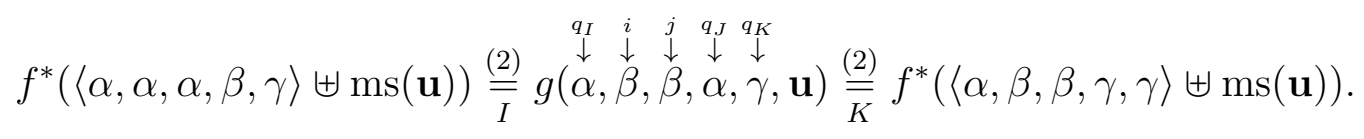

If $q_{K} \notin K \cup J$, then for any $\alpha, \beta, \gamma \in A$ and for any $\mathbf{u} \in A^{n-5}$,

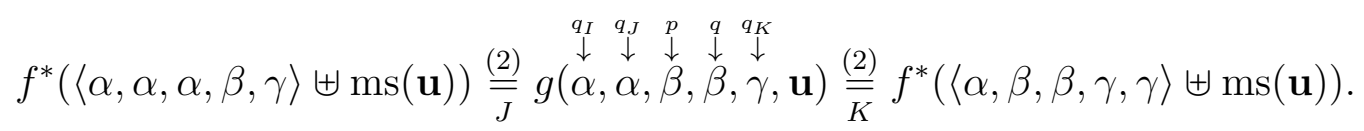

Thus, for all $\alpha, \beta, \gamma, \delta \in A$ and for all $\mathbf{u} \in A^{n-6}$, it holds that

$$
\begin{aligned}
f^{*}(\langle\alpha, \alpha, \alpha, \beta, \gamma, \delta\rangle \uplus \operatorname{ms}(\mathbf{u}))=f^{*}(\langle\alpha, \alpha, \beta, \gamma, \delta, \delta\rangle \uplus \operatorname{ms}(\mathbf{u})) & = \\
& f^{*}(\langle\alpha, \beta, \beta, \gamma, \gamma, \delta\rangle \uplus \operatorname{ms}(\mathbf{u})) .
\end{aligned}
$$

Let $S \in \mathcal{M}_{n}(A)$, let $E:=\operatorname{set}(S)$, and fix an element $e \in E$. Let $F$ be the multiset on $A$ given by the multiplicity function

$$
\mathbf{1}_{F}(x)= \begin{cases}|E|-n+1, & \text { if } x=e \\ 1, & \text { if } x \in E \backslash\{e\} \\ 0, & \text { if } x \notin E\end{cases}
$$

We will construct a sequence $S=S_{0}, S_{1}, \ldots, S_{r}=F(r \geqslant 1)$ of multisets in $\mathcal{M}_{n}(A)$ that satisfy $f^{*}\left(S_{i}\right)=f^{*}\left(S_{i-1}\right)$ for all $i \in[r]$. Let $S_{0}:=S$, and define $S_{1}$ by the following rules. 
- If $\mathbf{1}_{S}(e)>1$, then let $S_{1}:=S_{0}$. In this case obviously $f^{*}\left(S_{0}\right)=f^{*}\left(S_{1}\right)$.

- If $\mathbf{1}_{S}(e)=1$ and there exists $a \in A$ such that $\mathbf{1}_{S}(a) \geqslant 3$, then let $S_{1}:=S_{0} \uplus\langle e\rangle \backslash\langle a\rangle$. Then $f^{*}\left(S_{0}\right)=f^{*}\left(S_{1}\right)$ by (3) (take $\alpha=a$ and $\delta=e$, and consider the first and second expression in (3)).

- Otherwise we have that $\mathbf{1}_{S}(e)=1$ and, since $n \geqslant|A|+2$, there exist distinct elements $a$ or $b$ of $A$ such that $\mathbf{1}_{S}(a)=\mathbf{1}_{S}(b)=2$. Let $S_{1}:=S_{0} \uplus\langle e, e\rangle \backslash\langle a, b\rangle$. Then $f^{*}\left(S_{0}\right)=f^{*}\left(S_{1}\right)$ by $(3)$ (take $\alpha=e, \beta=a$, and $\gamma=b$, and consider the first and third expression in (3)).

Thus $S_{1}$ is a multiset with $\mathbf{1}_{S_{1}}(e)>1$. We proceed by the following recursion.

- If $i \geqslant 1$ and there exists $a \in A$ such that $\mathbf{1}_{S_{i}}(a)>1$ and $a \neq e$, then let $S_{i+1}:=$ $S_{i} \uplus\langle e\rangle \backslash\langle a\rangle$. Then $f^{*}\left(S_{i}\right)=f^{*}\left(S_{i+1}\right)$ by (3) (take $\alpha=e$ and $\delta=a$, and consider the first and second expression in (3)). Furthermore, $\mathbf{1}_{S_{i+1}}(e)>1$, and we can apply the recursive step again.

- Otherwise $S_{i}=U$, and we let $r:=i$ and stop the recursion.

The recursion will stop after a finite number of steps, and we have that $f^{*}(S)=f^{*}\left(S_{0}\right)=$ $f^{*}\left(S_{1}\right)=\cdots=f^{*}\left(S_{r}\right)=f^{*}(F)$. We conclude that if $S, T \in \mathcal{M}_{N}(A)$ are multisets such that $\operatorname{set}(S)=\operatorname{set}(T)$, then we have $f^{*}(S)=f^{*}(F)=f^{*}(T)$, as claimed.

Case 2: $q_{I}, q_{J} \notin I \cup J, q_{I}=q_{J}$. In this case $n \geqslant 5$. We have for any $\alpha, \beta, \gamma \in A$ and for any $\mathbf{u} \in A^{n-5}$ that

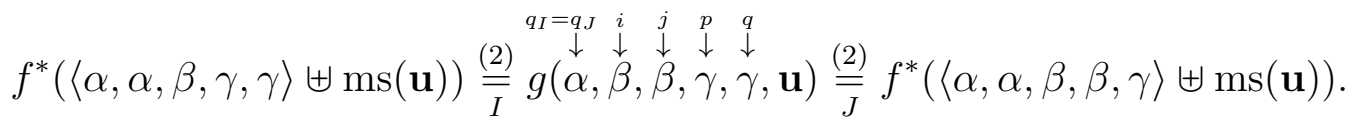

Let $K=\left\{q_{I}, p\right\}$. If $q_{K} \in K$, then for all $\alpha, \beta \in A$ and for all $\mathbf{u} \in A^{n-4}$,

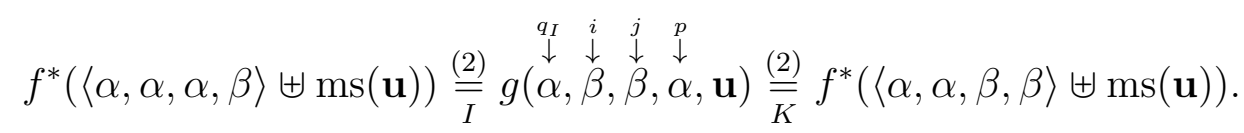

If $q_{K} \notin J \cup K$, then for all $\alpha, \beta \in A$ and for all $\mathbf{u} \in A^{n-4}$,

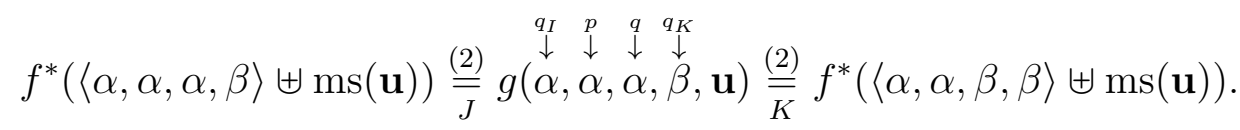

If $q_{K} \notin I \cup K$, then for all $\alpha, \beta, \gamma \in A$ and for all $\mathbf{u} \in A^{n-5}$,

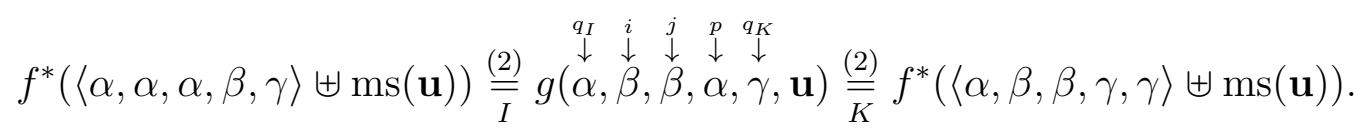

Thus, for all $\alpha, \beta, \gamma \in A$ and for all $\mathbf{u} \in A^{n-5}$, it holds that

$$
\begin{aligned}
f^{*}(\langle\alpha, \alpha, \alpha, \beta, \gamma\rangle \uplus \operatorname{ms}(\mathbf{u}))= & f^{*}(\langle\alpha, \alpha, \beta, \beta, \gamma\rangle \uplus \operatorname{ms}(\mathbf{u}))= \\
& f^{*}(\langle\alpha, \alpha, \beta, \gamma, \gamma\rangle \uplus \operatorname{ms}(\mathbf{u}))=f^{*}(\langle\alpha, \beta, \beta, \gamma, \gamma\rangle \uplus \operatorname{ms}(\mathbf{u})) .
\end{aligned}
$$

Proceeding in a similar way as in Case 1, we can show that the above identities imply that $f^{*}(S)=f^{*}(T)$ for all $S, T \in \mathcal{M}_{n}(A)$ such that $\operatorname{set}(S)=\operatorname{set}(T)$. 
Case 3: $q_{I} \notin I \cup J, q_{J} \in I$. In this case $n \geqslant 5$. We have for any $\alpha, \beta, \gamma \in A$ and for any $\mathbf{u} \in A^{n-5}$ that

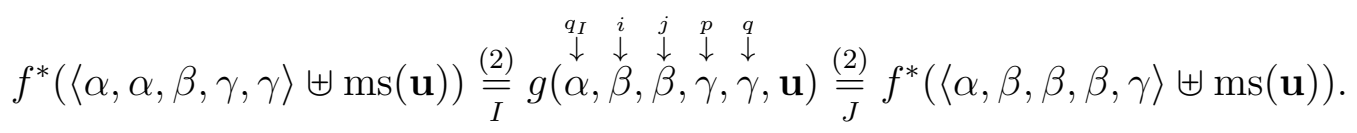

Let $K=\left\{q_{I}, p\right\}$. If $q_{K} \in K$, then for all $\alpha, \beta, \gamma \in A$ and for all $\mathbf{u} \in A^{n-5}$,

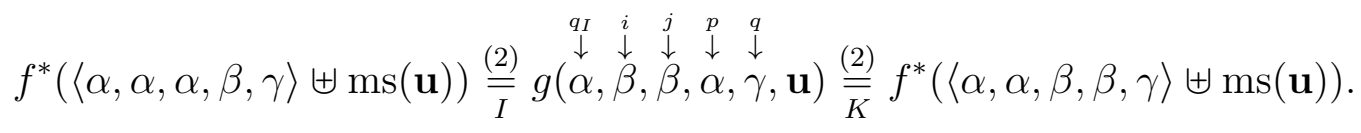

If $q_{K} \in I$, then for all $\alpha, \beta, \gamma \in A$ and for all $\mathbf{u} \in A^{n-5}$,

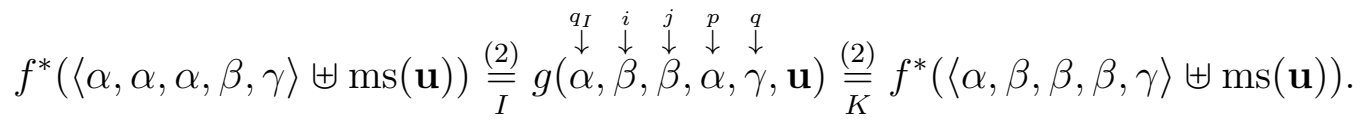

If $q_{K}=q$, then for all $\alpha, \beta, \gamma \in A$ and for all $\mathbf{u} \in A^{n-5}$,

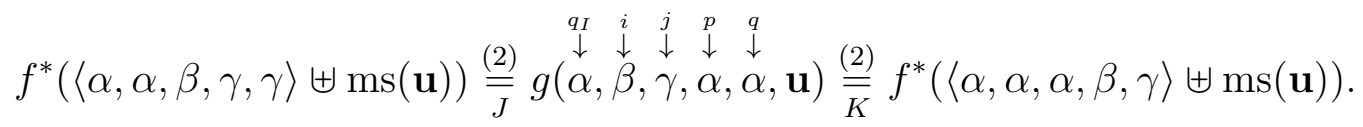

If $q_{K} \notin I \cup J \cup\left\{q_{I}\right\}$, then for all $\alpha, \beta, \gamma \in A$ and for all $\mathbf{u} \in A^{n-5}$,

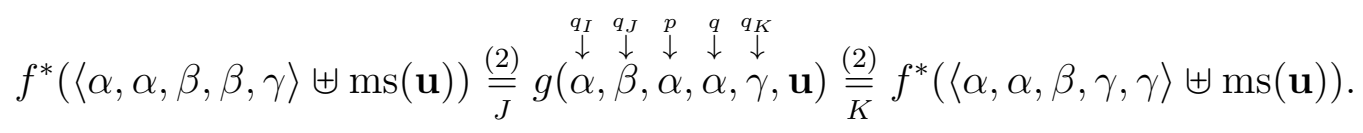

Thus, for all $\alpha, \beta, \gamma \in A$ and for all $\mathbf{u} \in A^{n-5}$, it holds that

$$
\begin{aligned}
& f^{*}(\langle\alpha, \alpha, \alpha, \beta, \gamma\rangle \uplus \operatorname{ms}(\mathbf{u}))=f^{*}(\langle\alpha, \beta, \beta, \beta, \gamma\rangle \uplus \operatorname{ms}(\mathbf{u}))= \\
& f^{*}(\langle\alpha, \beta, \gamma, \gamma, \gamma\rangle \uplus \operatorname{ms}(\mathbf{u}))=f^{*}(\langle\alpha, \alpha, \beta, \beta, \gamma\rangle \uplus \operatorname{ms}(\mathbf{u}))= \\
& f^{*}(\langle\alpha, \alpha, \beta, \gamma, \gamma\rangle \uplus \operatorname{ms}(\mathbf{u}))=f^{*}(\langle\alpha, \beta, \beta, \gamma, \gamma\rangle \uplus \operatorname{ms}(\mathbf{u})) .
\end{aligned}
$$

As in the previous cases, we can show that $f^{*}(S)=f^{*}(T)$ for all $S, T \in \mathcal{M}_{n}(A)$ such that $\operatorname{set}(S)=\operatorname{set}(T)$.

Case 4: $q_{I} \in J, q_{J} \in I$. Without loss of generality, we may assume that $q_{I}=p$ and $q_{J}=j$. We have for any $\alpha, \beta \in A$ and for any $\mathbf{u} \in A^{n-4}$,

$$
f^{*}(\langle\alpha, \beta, \beta, \beta\rangle \uplus \operatorname{ms}(\mathbf{u})) \stackrel{(2)}{=} g\left(\begin{array}{cccc}
i & j & p & q \\
I & \downarrow & \downarrow & \downarrow \\
\alpha & \alpha & \alpha & \beta
\end{array}, \stackrel{(2)}{\beta}, \mathbf{u}\right) \underset{J}{=} f^{*}(\langle\alpha, \alpha, \alpha, \beta\rangle \uplus \operatorname{ms}(\mathbf{u})) .
$$

Let $K=\{i, q\}$. If $q_{K} \in\{i, j, q\}$, then for all $\alpha, \beta \in A$ and for all $\mathbf{u} \in A^{n-4}$,

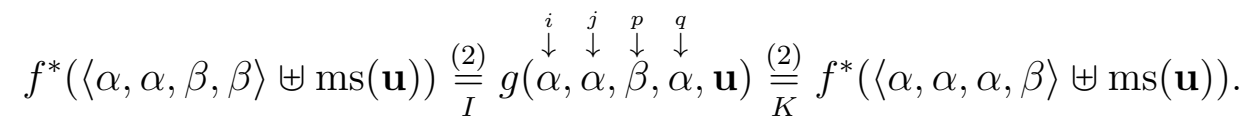


If $q_{K} \in\{i, p, q\}$, then for all $\alpha, \beta \in A$ and for all $\mathbf{u} \in A^{n-4}$,

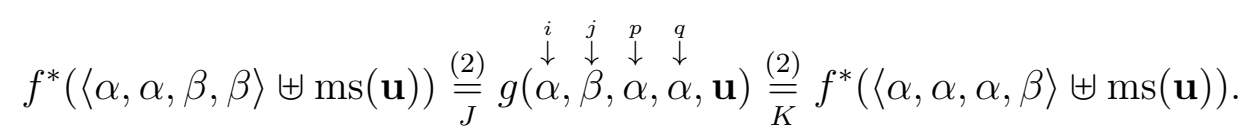

If $q_{K} \notin\{i, j, p, q\}$, then $n \geqslant 5$. Let $L=\left\{q_{K}, i\right\}$. If $q_{L} \in\left\{q_{K}, i, j\right\}$, then for all $\alpha, \beta, \gamma \in A$ and for all $\mathbf{u} \in A^{n-5}$,

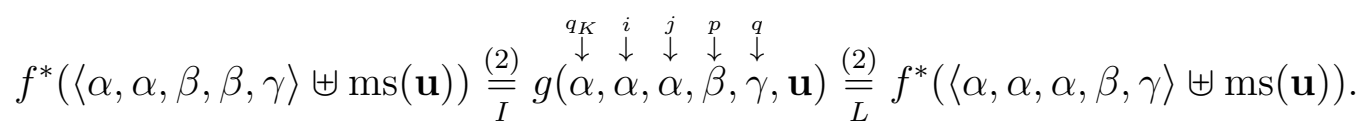

If $q_{L} \in\{p, q\}$, then for all $\alpha, \beta, \gamma \in A$ and for all $\mathbf{u} \in A^{n-5}$,

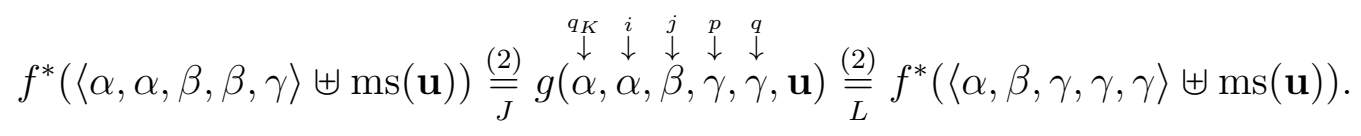

If $q_{L} \notin\{i, j, p, q\}$, then for all $\alpha, \beta \in A$ and for all $\mathbf{u} \in A^{n-4}$,

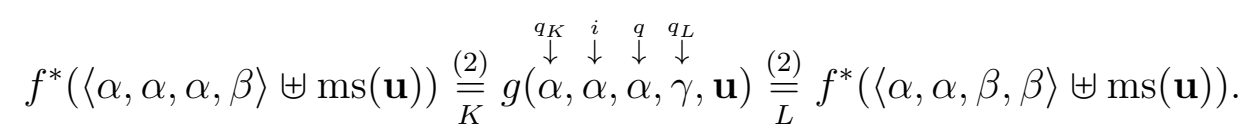

Thus, for all $\alpha, \beta \in A$ and for all $\mathbf{u} \in A^{n-4}$, it holds that

$$
f^{*}(\langle\alpha, \alpha, \alpha, \beta\rangle \uplus \operatorname{ms}(\mathbf{u}))=f^{*}(\langle\alpha, \alpha, \beta, \beta\rangle \uplus \operatorname{ms}(\mathbf{u}))=f^{*}(\langle\alpha, \beta, \beta, \beta\rangle \uplus \operatorname{ms}(\mathbf{u})) .
$$

As in the previous cases, we can show that $f^{*}(S)=f^{*}(T)$ for all $S, T \in \mathcal{M}_{n}(A)$ such that $\operatorname{set}(S)=\operatorname{set}(T)$.

Cases 1-4 exhaust all possibilities, and the proof of the claim is complete.

Claim 5.1.2. If there exist $I, J \in\left(\begin{array}{c}{[n]} \\ 2\end{array}\right)$ such that $I \cap J=\emptyset$ and $q_{I}, q_{J} \in J$, then $f^{*}(S)=$ $f^{*}(T)$ for all $S, T \in \mathcal{M}_{n}(A)$ such that $\operatorname{set}(S)=\operatorname{set}(T)$.

Proof of Claim 5.1.2. Let $I=\{i, j\}, J=\{p, q\}$, and assume that $I \cap J=\emptyset$ and $q_{I}, q_{J} \in J$. Then for any $\alpha, \beta \in A$ and for any $\mathbf{u} \in A^{n-4}$ we have

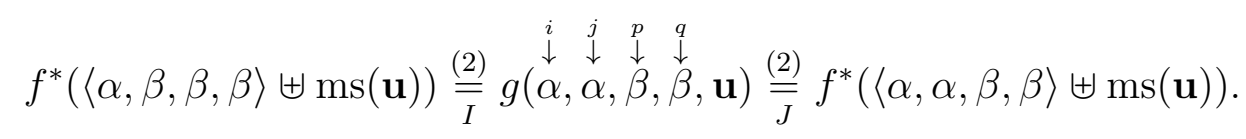

Proceeding as we did in the proof of Claim 5.1.1, we can show that $f^{*}(S)=f^{*}(T)$ for all $S, T \in \mathcal{M}_{n}(A)$ such that $\operatorname{set}(S)=\operatorname{set}(T)$.

Claim 5.1.3. If there exists $I \in\left(\begin{array}{c}{[n]} \\ 2\end{array}\right)$ such that $q_{I} \notin I$, then $f^{*}(S)=f^{*}(T)$ for all $S, T \in \mathcal{M}_{n}(A)$ such that $\operatorname{set}(S)=\operatorname{set}(T)$.

Proof of Claim 5.1.3. Assume that $I \in\left(\begin{array}{c}{[n]} \\ 2\end{array}\right)$ is such that $q_{I} \notin I$. Since $n \geqslant 4$, there exists $p \in[n] \backslash\left(I \cup\left\{q_{I}\right\}\right)$. Let $J=\left\{p, q_{I}\right\}$. Depending on whether $q_{J} \notin J$ or $q_{J} \in J$, either Claim 5.1.1 or Claim 5.1.2 implies that $f^{*}(S)=f^{*}(T)$ for all $S, T \in \mathcal{M}_{n}(A)$ such that $\operatorname{set}(S)=\operatorname{set}(T)$. 
(Proof of Theorem 5.1 continued) If there exists $I \in\left(\begin{array}{c}{[n]} \\ 2\end{array}\right)$ such that $q_{I} \notin I$, then by Claim 5.1.3, we have that $f^{*}(S)=f^{*}(T)$ for all $S, T \in \mathcal{M}_{n}(A)$ such that $\operatorname{set}(S)=\operatorname{set}(T)$. Then $f$ is determined by supp, and it is reconstructible by Proposition 3.8. Otherwise $q_{I} \in I$ for all $I \in\left(\begin{array}{c}{[n]} \\ 2\end{array}\right)$, and we have for every a $\in A^{n}$ that if $a_{\min I}=a_{\max I}$ for some $I \in\left(\begin{array}{c}{[n]} \\ 2\end{array}\right)$, then $a_{q_{I}}=a_{\min I}=a_{\max I}$. Then Equation (2) yields $g(\mathbf{a})=f^{*}\left(\operatorname{ms}(\mathbf{a}) \backslash\left\langle a_{\max I}\right\rangle \uplus\right.$ $\left.\left\langle a_{q_{I}}\right\rangle\right)=f^{*}(\operatorname{ms}(\mathbf{a}))$ for all $\mathbf{a} \in A^{n}$, i.e., $g=f$. We conclude that $f$ is reconstructible.

Proposition 5.2. Assume that $n>\max (k, 3)$ and $f, g: A^{n} \rightarrow B$ are totally symmetric. If $\operatorname{deck} f=\operatorname{deck} g$, then $f=g$.

Proof. Proposition 4.3 and the assumption that $\operatorname{deck} f=\operatorname{deck} g$ imply that $f_{I} \equiv g_{J}$ for all $I, J \in\left(\begin{array}{c}{[n]} \\ 2\end{array}\right)$. In particular, setting $N:=\{n-1, n\}$, we have that $f_{N} \equiv g_{N}$; hence there exists a permutation $\tau \in \Sigma_{n-1}$ such that $f_{N}(\mathbf{a})=g_{N}(\mathbf{a} \tau)$ for all $\mathbf{a} \in A^{n-1}$. By the definition of identification minor, we have

$$
f\left(\mathbf{a} \delta_{N}\right)=f_{N}(\mathbf{a})=g_{N}(\mathbf{a} \tau)=g\left(\mathbf{a} \tau \delta_{N}\right)
$$

for all $\mathbf{a} \in A^{n-1}$.

We want to show that $f=g$, that is $f(\mathbf{a})=g(\mathbf{a})$ for all $\mathbf{a} \in A^{n}$. Let $\mathbf{a} \in A^{n}$ be arbitrary. Since $n>k$, there is an element $\alpha \in A$ that has at least two occurrences in $\mathbf{a}$. By the total symmetry of $f$ and $g$, we may assume that the last two components of a are equal to $\alpha$, i.e., $a_{n-1}=a_{n}=\alpha$. Let $\mathbf{b}$ be the $(n-1)$-tuple that is obtained by removing the last entry from $\mathbf{a}$. We clearly have $\mathbf{a}=\mathbf{b} \delta_{N}$.

We need to distinguish between two cases depending on whether $\tau(n-1)=n-1$ or not. Consider first the case that $\tau(n-1)=n-1$. By Equation (4) and the total symmetry (TS) of $g$ we have

$$
f(\mathbf{a})=f\left(\mathbf{b} \delta_{N}\right) \stackrel{(4)}{=} g\left(\mathbf{b} \tau \delta_{N}\right) \stackrel{\text { TS }}{=} g(\mathbf{a}) .
$$

Consider then the case that $\tau(n-1)=r \neq n-1$. Fix an element $s$ of $[n-1] \backslash\{r, n-1\}$; this set is nonempty since $n>3$. Let $\beta:=a_{r}, \gamma:=a_{s}$. Repeated applications of (4) and the total symmetry of $f$ and $g$ yield

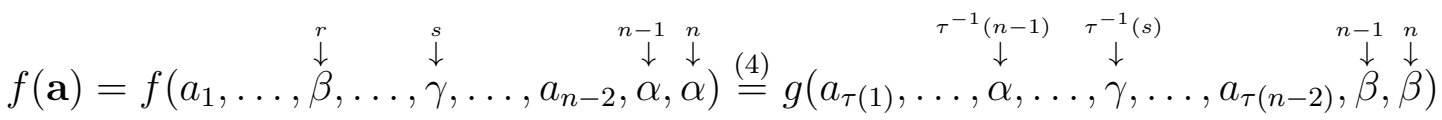

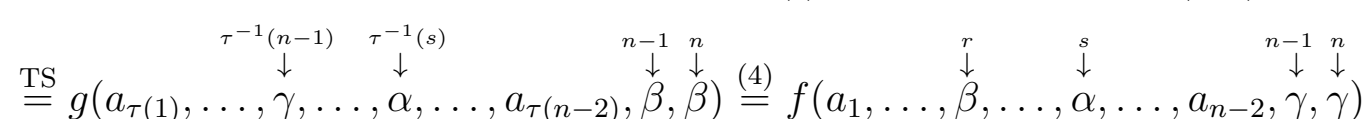

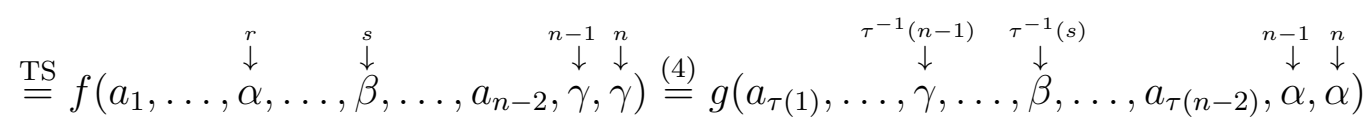

$$
\begin{aligned}
& \stackrel{\text { TS }}{=} g\left(a_{1}, \ldots, \stackrel{r}{\downarrow}, \ldots, \stackrel{s}{\downarrow}, \ldots, a_{n-2}, \stackrel{n-1}{\downarrow}, \stackrel{n}{\alpha}, \stackrel{\downarrow}{\alpha}\right)=g(\mathbf{a}) .
\end{aligned}
$$

This shows that $f(\mathbf{a})=g(\mathbf{a})$ for all $\mathbf{a} \in A^{n}$, i.e., $f=g$. 


\section{Reconstruction problems for hypergraphs}

We conclude this paper with a brief discussion of how our reconstruction problem for functions and identification minors can be interpreted in terms of hypergraphs. In the special case of Boolean functions, the problem translates straightforwardly into certain reconstruction problems for hypergraphs.

The $n$-ary Boolean functions are in one-to-one correspondence with the hypergraphs with vertex set $[n]$. Perhaps the most natural correspondence is obtained by associating to each $n$-ary Boolean function $f$ a hypergraph on $[n]$ whose edges correspond to the true points of $f$. In precise terms, for a Boolean function $f:\{0,1\}^{n} \rightarrow\{0,1\}$, define the hypergraph $G_{f}^{\text {true }}=\left(V_{f}, E_{f}^{\text {true }}\right)$, where $V_{f}:=[n]$ and for every $S \subseteq V_{f}$ it holds that $S \in E_{f}^{\text {true }}$ if and only if $f\left(\mathbf{e}_{S}\right)=1$. Here $\mathbf{e}_{S}$ denotes the characteristic tuple of $S \subseteq[n]$, i.e., the unique $n$-tuple $\left(e_{1}, \ldots, e_{n}\right) \in\{0,1\}^{n}$ satisfying $e_{i}=1$ if and only if $i \in S$.

For a hypergraph $G=(V, E)$ with $V=[n]$ and for $I \in\left(\begin{array}{c}{[n]} \\ 2\end{array}\right)$, define the hypergraph $G_{I}^{-}=\left(V_{I}^{-}, E_{I}^{-}\right)$with $V_{I}^{-}=[n] \backslash I \cup\{v\}$, where $v$ is a new element not in $[n]$, and

$$
E_{I}^{-}:=\{S: S \in E, S \cap I=\emptyset\} \cup\{S \backslash I \cup\{v\}: S \in E, I \subseteq S\} .
$$

In other words, if $I=\{i, j\}$, then the operation of forming $G_{I}^{-}$from $G$ performs the following: vertices $i$ and $j$ are contracted into a new vertex $v$, every edge that contains $i$ or $j$ but not both is deleted, and each edge $S$ that contains both $i$ and $j$ is replaced by $S \backslash I \cup\{v\}$.

We can now define the deck of a hypergraph $G$ on vertex set $[n]$ as the multiset $\left\langle G_{I}^{-} / \cong: I \in\left(\begin{array}{c}{[n]} \\ 2\end{array}\right)\right\rangle$ of isomorphism types of the hypergraphs $G_{I}^{-}$. It is straightforward to verify that $G_{f_{I}}^{\text {true }}$ is isomorphic to $\left(G_{f}^{\text {true }}\right)_{I}^{-}$for any Boolean function $f:\{0,1\}^{n} \rightarrow\{0,1\}$ and for any $I \in\left(\begin{array}{c}{[n]} \\ 2\end{array}\right)$. Therefore, the reconstruction problem for hypergraphs $G$ and derived hypergraphs $G_{I}^{-}$is essentially the same as the reconstruction problem for Boolean functions and identification minors.

Another natural translation between Boolean functions and hypergraphs was proposed by Bouaziz, Couceiro and Pouzet in [6]. It is well known that every Boolean function is represented by a unique multilinear polynomial over the two-element field GF(2), i.e., a polynomial in which each variable in each monomial has degree at most one (see Reed [31], Muller [27] or Zhegalkin [36]). We can then associate to each $n$-ary Boolean function $f$ a hypergraph on $[n]$ whose edges correspond to the monomials of the polynomial representation of $f$. In precise terms, if $f:\{0,1\}^{n} \rightarrow\{0,1\}$ is given by

$$
f\left(x_{1}, \ldots, x_{n}\right)=\sum_{S \in \mathcal{S}} \prod_{i \in S} x_{i}
$$

for some $\mathcal{S} \subseteq \mathcal{P}([n])$ (such a set system $\mathcal{S}$ exists and is unique), then we define the hypergraph $G_{f}^{\text {poly }}=\left(V_{f}, E_{f}^{\text {poly }}\right)$ with $V_{f}:=[n]$ and $E_{f}^{\text {poly }}:=\mathcal{S}$.

For a hypergraph $G=(V, E)$ with $V=[n]$ and for $I \in\left(\begin{array}{c}{[n]} \\ 2\end{array}\right)$, define the hypergraph $G_{I}^{+}=\left(V_{I}^{+}, E_{I}^{+}\right)$with $V_{I}^{+}=[n] \backslash I \cup\{v\}$, where $v$ is a new element not in $[n]$, and for each $S \subseteq V_{I}^{+}$, we have $S \in E_{I}^{+}$if and only if either 
(i) $v \notin S$ and $S \in E$, or

(ii) $v \in S$ and $|\{T \in E: T \cap I \neq \emptyset \& T \backslash I=S \backslash\{v\}\}|$ is odd.

In other words, if $I=\{i, j\}$, then the operation of forming $G_{I}^{+}$from $G$ performs the following: vertices $i$ and $j$ are contracted into a new vertex $v$, edges containing $i$ or $j$ are deleted, and for each $S \subseteq V \backslash I$, the set $S \cup\{v\}$ is taken as an edge of $G_{I}^{+}$if and only if exactly one or three of the sets $S \cup\{i\}, S \cup\{j\}$ and $S \cup\{i, j\}$ are edges of $G$.

We can now define the deck of a hypergraph $G$ on vertex set $[n]$ as the multiset $\left\langle G_{I}^{+} / \cong: I \in\left(\begin{array}{c}{[n]} \\ 2\end{array}\right)\right\rangle$ of isomorphism types of the hypergraphs $G_{I}^{+}$. It is easy to verify, and it was shown in [6], that $G_{f_{I}}^{\text {poly }}$ is isomorphic to $\left(G_{f}^{\text {poly }}\right)_{I}^{+}$for any Boolean function $f:\{0,1\}^{n} \rightarrow\{0,1\}$ and for any $I \in\left(\begin{array}{c}{[n]} \\ 2\end{array}\right)$. Therefore, the reconstruction problem for hypergraphs $G$ and derived hypergraphs $G_{I}^{+}$is essentially the same as the reconstruction problem for Boolean functions and identification minors.

The two reconstruction problems for hypergraphs described above do not seem to be related to the reconstruction problems for graphs or hypergraphs that were mentioned in Section 1, because the cards are formed in rather different ways. They might, however, be of interest on their own right, due to the connection to the reconstruction problem for functions and identification minors. It would perhaps be worthwhile investigating the reconstructibility of some subclasses of hypergraphs. A particularly important and natural subclass of hypergraphs is that of graphs.

Problem 6.1. Which graphs $G$ on $[n]$ are uniquely determined, up to isomorphism, by the deck $\left\langle G_{I}^{-} / \cong: I \in\left(\begin{array}{c}{[n]} \\ 2\end{array}\right)\right\rangle$ ? Which ones are determined by the deck $\left\langle G_{I}^{+} / \cong: I \in\left(\begin{array}{c}{[n]} \\ 2\end{array}\right)\right\rangle$ ?

\section{Acknowledgments}

I would like to thank Miguel Couceiro, Karsten Schölzel, and Tamás Waldhauser for many inspiring discussions on minors of functions, reconstruction problems, and permutations. I am also grateful to the anonymous referee for constructive remarks and valuable suggestions that greatly helped improve this paper.

\section{References}

[1] L. Babai. Automorphism groups, isomorphism, reconstruction. In: R. L. Graham, M. Grötschel, and L. Lovász (eds.), Handbook of Combinatorics, Vol. 2, pages 14471540. Elsevier, Amsterdam, 1995.

[2] J. Berman and A. Kisielewicz. On the number of operations in a clone. Proc. Amer. Math. Soc. 122 (1994) 359-369.

[3] J. A. Bondy. A graph reconstructor's manual. In: A. D. Keedwell (ed.), Surveys in Combinatorics, 1991, volume 166 of London Math. Soc. Lecture Note Ser., pages 221-252. Cambridge Univ. Press, Cambridge, 1991.

[4] J. A. Bondy and R. L. Hemminger. Graph reconstruction-a survey. J. Graph Theory 1 (1977) 227-268. 
[5] J. A. Bondy and U. S. R. Murty. Graph Theory. Graduate Texts in Mathematics, no. 244. Springer, 2008.

[6] M. Bouaziz, M. Couceiro, and M. Pouzet. Join-irreducible Boolean functions. Order 27 (2010) 261-282.

[7] T. H. Brylawski. Reconstructing combinatorial geometries. In: R. A. Bari and F. Harary (eds.), Graphs and Combinatorics, volume 406 of Lecture Notes in Math., pages 226-235. Springer, 1974.

[8] T. H. Brylawski. On the nonreconstructibility of combinatorial geometries. J. Combin. Theory Ser. B 19 (1975) 72-76.

[9] M. Couceiro and S. Foldes. On closed sets of relational constraints and classes of functions closed under variable substitutions. Algebra Universalis 54 (2005) 149-165.

[10] M. Couceiro and E. Lehtonen. Generalizations of Świerczkowski's lemma and the arity gap of finite functions. Discrete Math. 309 (2009) 5905-5912.

[11] K. Denecke and S. L. Wismath. Universal Algebra and Applications in Theoretical Computer Science. Chapman \& Hall/CRC, Boca Raton, 2002.

[12] O. Ekin, S. Foldes, P. L. Hammer, and L. Hellerstein. Equational characterizations of Boolean function classes. Discrete Math. 211 (2000) 27-51.

[13] M. N. Ellingham. Recent progress in edge-reconstruction. Seventeenth Manitoba Conference on Numerical Mathematics and Computing, Congr. Numer. 62 (1988) $3-20$.

[14] R. Fraïssé. Abritement entre relations et spécialement entre chaînes. In volume V of Symposia Mathematica, pages 203-251. Academic Press, London, 1971.

[15] F. Harary. On the reconstruction of a graph from a collection of subgraphs. In Theory of Graphs and Its Applications (Proc. Sympos. Smolenice, 1963), pages 47-52. Publ. House Czechoslovak Acad. Sci., Prague, 1964.

[16] F. Harary. A survey on the reconstruction conjecture, In: R. A. Bari and F. Harary (eds.), Graphs and Combinatorics, volume 406 of Lecture Notes in Math., pages 1828. Springer, 1974.

[17] P. J. Kelly. On Isometric Transformations, Ph.D. thesis. University of Wisconsin, 1942.

[18] P. J. Kelly. A congruence theorem for trees. Pacific J. Math. 7 (1957) 961-968.

[19] W. L. Kocay. A family of nonreconstructible hypergraphs. J. Combin. Theory Ser. B 42 (1987) 46-63.

[20] D. Lau. Function Algebras on Finite Sets. Springer, Berlin, 2006.

[21] E. Lehtonen. Descending chains and antichains of the unary, linear, and monotone subfunction relations. Order 23 (2006) 129-142.

[22] E. Lehtonen and Á. Szendrei. Equivalence of operations with respect to discriminator clones. Discrete Math. 309 (2009) 673-685. 
[23] B. Manvel. Reconstruction of graphs: progress and prospects. 250th Anniversary Conference on Graph Theory, Congr. Numer. 63 (1988) 177-187.

[24] B. Manvel and P. K. Stockmeyer. On reconstruction of matrices. Math. Mag. 44 (1971) 218-221.

[25] B. D. McKay. Small graphs are reconstructible. Australas. J. Combin. 15 (1997) 123-126.

[26] M. Monks. The solution to the partition reconstruction problem. J. Combin. Theory Ser. A 116 (2009) 76-91.

[27] D. E. Muller. Application of Boolean algebra to switching circuit design and to error correction. IRE Trans. Electron. Comput. 3(3) (1954) 6-12.

[28] C. St. J. A. Nash-Williams. The reconstruction problem. In: L. W. Beinecke and R. J. Wilson (eds.), Selected Topics in Graph Theory, pages 205-236. Academic Press, London, 1978.

[29] N. Pippenger. Galois theory for minors of finite functions. Discrete Math. 254 (2002) 405-419.

[30] J.-X. Rampon. What is reconstruction for ordered sets? Discrete Math. 291 (2005) $191-233$.

[31] I. S. Reed. A class of multiple-error-correcting codes and the decoding scheme. IRE Trans. Inf. Theory 4(4) (1954) 38-49.

[32] P. K. Stockmeyer. A census of nonreconstructible digraphs. I. Six related families. J. Combin. Theory Ser. B 31 (1981) 232-239.

[33] Á. Szendrei. Clones in Universal Algebra. Séminaire de mathématiques supérieures, no. 99. Les Presses de l'Université de Montréal, Montréal, 1986.

[34] S. M. Ulam. A Collection of Mathematical Problems. Interscience Tracts in Pure and Applied Mathematics, no. 8. Interscience Publishers, New York-London, 1960.

[35] R. Willard. Essential arities of term operations in finite algebras. Discrete Math. 149 (1996) 239-259.

[36] I. I. Zhegalkin. On the calculation of propositions in symbolic logic. Mat. Sb. 34 (1927) 9-28 (in Russian).

[37] I. E. Zverovich. Characterizations of closed classes of Boolean functions in terms of forbidden subfunctions and Post classes. Discrete Appl. Math. 149 (2005) 200-218. 